\title{
Unfitted hybrid high-order methods for the wave equation
}

\author{
Erik Burman* $\quad$ Omar Duran $^{\dagger} \quad$ Alexandre Ern ${ }^{\ddagger}$
}

November 10, 2021

\begin{abstract}
We design an unfitted hybrid high-order (HHO) method for the wave equation. The wave propagates in a domain where a curved interface separates subdomains with different material properties. The key feature of the discretization method is that the interface can cut more or less arbitrarily through the mesh cells. We address both the second-order formulation in time of the wave equation and its reformulation as a first-order system. We prove $H^{1}$-error estimates for a space semi-discretization in space of the second-order formulation, leading to optimal convergence rates for smooth solutions. Numerical experiments illustrate the theoretical findings and show that the proposed numerical schemes can be used to simulate accurately the propagation of acoustic waves in heterogeneous media, with meshes that are not fitted to the geometry. For the second-order formulation, the implicit, second-order accurate Newmark scheme is used for the time discretization, whereas (diagonally-implicit or explicit) Runge-Kutta schemes up to fourth-order accuracy are used for the first-order formulation. In the explicit case, we study the CFL condition on the time step and observe that the unfitted approach combined with local cell agglomeration leads to a comparable condition as when using fitted meshes.
\end{abstract}

Mathematics Subjects Classification. 65M12, 65M60, 74J10, 74S05.

Keywords. Hybrid high-order methods, unfitted mesh, error analysis, wave equation, heterogeneous media

\section{Introduction}

The accurate numerical approximation of wave phenomena is important in many applications. In particular, in medical imagining problems such as photo acoustic tomography, or geophysical applications involving seismic waves, it is paramount to be able to accurately approximate wave propagation through heterogeneous materials in domains with complex geometry. Two examples from geophysics are solid layers with varying wave speed on the one hand and solid domains with fluid inclusions on the other hand. It is also important to be able to handle problems where the ratio between the characteristic length scale of the domain and the wave length is large, which is typically the case for high frequency problems in medical applications or the propagation of seismic waves over large distances. Moreover, the geometry can be given by measurements, have curved boundaries or even be an unknown of the problem. These design

\footnotetext{
${ }^{*}$ Department of Mathematics, University College London, London, UK-WC1E 6BT, UK. E-mail address: e.burman@ucl.ac.uk

${ }^{\dagger}$ CERMICS, Ecole des Ponts, 77455 Marne la Vallée cedex 2, and INRIA Paris, 75589 Paris, France. E-mail address: omar.duran@enpc.fr

${ }^{\ddagger}$ CERMICS, Ecole des Ponts, 77455 Marne la Vallée cedex 2, and INRIA Paris, 75589 Paris, France. E-mail address: alexandre.ern@enpc.fr
} 
constraints indicate the need for a method that can handle heterogeneous material parameters (or models), propagates waves with low dissipation and dispersion, and is flexible for the integration of complex geometries.

To obtain a method that meets the above requirements, we herein combine the unfitted hybrid high-order (HHO) method introduced in [10] for elliptic interface problems and further developed in [6] with the HHO methods for wave equations recently proposed and studied numerically in [8] and analyzed in the space semi-discrete case in [9]. The high-order approximation of the method allows for good accuracy and low dispersion, while the unfitted character allows for great flexibility in the integration of interface geometries. In the context of stationary elliptic interface problems, the method can handle heterogeneous physical parameters and is robust for high-contrast problems. In this work, we handle the heterogeneous setting but do not explore the robustness with respect to high contrast. Finally, static condensation allows for an efficient numerical solution of the system by the local elimination of all the unknowns attached to the mesh cells.

HHO methods were introduced in [18] for linear diffusion problems and in [17] for locking-free linear elasticity. These methods rely on a pair of unknowns, combining polynomials attached to the mesh faces and to the mesh cells, and the cell unknowns can be eliminated locally by a static condensation procedure. HHO methods were bridged to hybridizable discontinuous Galerkin (HDG) methods and to nonconforming virtual element methods (ncVEM) in [13]. HHO methods also share similar devising principles with weak Galerkin (WG) methods [38]. In the unfitted HHO method, interfaces or boundaries are allowed to cut through the cells in a general fashion. The interface conditions are then integrated at the cell level. To avoid instabilities arising due to interface cell intersections resulting in very small or anisotropic sub-cells, a local cell-agglomeration algorithm has been designed in [6], drawing on earlier ideas from [25, 10]. Notice that the cell-agglomeration procedure crucially exploits the polyhedral features of $\mathrm{HHO}$ methods.

In the case of fitted meshes, HHO methods applied to the wave equation were devised and investigated numerically in [8], where in particular the significant improvement of solution quality for heterogeneous problems obtained by high-order approximation was showcased. The methods were analyzed in [9], leading to $H^{1}$-error estimates decaying with rate $\mathcal{O}\left(h^{k+1}\right)$ for smooth solutions and $L^{2}$-error estimates decaying with rate $\mathcal{O}\left(h^{k+2}\right)$ (assuming full elliptic regularity pickup). Here, $k$ denotes the polynomial order of the skeletal unknowns. Numerical investigations of the method applied to the elastic wave equations were also reported. In the present paper, we focus on the propagation of acoustic waves in heterogeneous media using unfitted meshes. Considering both the second-order scalar wave equation and its first-order system formulation, we design unfitted HHO methods. In the case of the second-order form with space semi-discretization, we propose an error analysis in the $H^{1}$-norm, drawing on the ideas of [9] for the fitted HHO method applied to the wave equation. The analysis shows that the unfitted HHO method has accuracy similar to the fitted version discussed above. In the numerical section, we show, by comparing with the fitted method, that the perturbation introduced by the unfitted geometry is moderate.

Many numerical methods exist for the semi-discretization in space of the wave equation. High-order continuous finite elements (cG) are reviewed, e.g., in [16]. Discontinuous Galerkin $(\mathrm{dG})$ methods have been successfully applied to the wave equation, written either as a first-order system [22, 28] or in its original second-order formulation in time [24]. Hybridizable dG (HDG) methods have been devised in [29, 32] for the first-order formulation, whereas the second-order formulation in time has been considered in [30, 14] with an eye toward conservation properties. The convergence analysis of HDG schemes has been performed in [15] in the time-continuous case and in [23] by considering a Petrov-Galerkin time discretization. The design of HDG methods for geophysical applications and with special focus on the coupling of acoustics and elastodynamics 
was considered in [37]. Unfitted methods for wave equations were first introduced in [33] using cG and the cutFEM framework [7], with ghost penalty stabilization [5] to ensure the robustness with respect to the interface position. The extension to high-order cG methods was considered in [34] and an extension to elastodynamics in [35]. A cut finite element method using cG and lumped mass explicit time stepping was proposed in [11] using discrete extension operators that generalize the $\mathrm{cG}$ agglomeration procedure of [2]. The use of hybridized nonconforming unfitted methods is much less explored. Recently, a numerical study of the use of local time-stepping as an alternative to cell agglomeration in the context of cut hybrid discontinuous Galerkin methods (placing degrees of freedom on the boundary) was proposed in [31].

This paper is organized as follows. In Section 2, we present the model problem. In Section 3, we introduce the unfitted HHO method and the tools from $[10,6]$ that are used for its formulation and analysis. The application of the framework to the wave equation is considered in Section 4 for the second-order formulation in time and in Section 5 for the first-order formulation. In Section 6, we perform the error analysis, focusing for brevity on the second-order formulation. In Section 7, we discuss numerical results to verify the convergence rates predicted by the theory. We also study the integration for curved interfaces to ensure that the results are not polluted by under-resolved geometry. Then we consider different methods for the time discretization: the implicit, second-order accurate Newmark scheme for the second-order formulation and RungeKutta (RK) schemes up to fourth-order accuracy (diagonally-implicit or explicit) for the firstorder formulation. We compare the performances of the fitted and the unfitted methods on a model problem with a flat interface for which a semi-analytical solution is available at least over a certain time window. We then consider a curved interface and compare the results of the unfitted method using various time integration schemes, including a study of the CFL condition for the explicit RK schemes. Finally, we draw some conclusions in Section 8.

\section{Model problem}

Let $\Omega$ be a polyhedral domain in $\mathbb{R}^{d}, d \in\{2,3\}$ (open, bounded, connected, Lipschitz subset of $\left.\mathbb{R}^{d}\right)$ with boundary $\partial \Omega$. Let $J:=\left(0, T_{\mathrm{f}}\right)$ be the time interval with $T_{\mathrm{f}}>0$. We use standard notation for the Lebesgue, Sobolev, and Bochner-Sobolev spaces. Boldface notation is used for vectors and vector-valued fields. For a subset $S \subset \mathbb{R}^{d}$, we denote $(\cdot, \cdot)_{S}$ the $L^{2}(S)$-inner product with appropriate Lebesgue measure and $\|\cdot\|_{S}$ the induced norm. For a weight function $\phi \in L^{\infty}(\Omega)$ taking positive values uniformly bounded from below away from zero, we use the shorthand notation $\|\cdot\|_{\phi ; S}:=\left\|\phi^{\frac{1}{2}} \cdot\right\|_{S}$, together with a similar notation for vector-valued fields.

Consider a partition of $\Omega$ into two disjoint subdomains $\bar{\Omega}=\overline{\Omega_{1}} \cup \overline{\Omega_{2}}$ with the interface $\Gamma:=\partial \Omega_{1} \cap \partial \Omega_{2}$. The interface $\Gamma$ is assumed to be a smooth $(d-1)$-dimensional manifold of class $C^{2}$ that is not self-intersecting. Our goal is to approximate the solution $p: J \times\left(\Omega_{1} \cup \Omega_{2}\right) \rightarrow \mathbb{R}$ of the following acoustic wave propagation problem with interface:

$$
\begin{aligned}
\frac{1}{\kappa} \partial_{t t} p-\nabla \cdot\left(\frac{1}{\rho} \nabla p\right) & =f & & \text { in } J \times\left(\Omega_{1} \cup \Omega_{2}\right), \\
\llbracket p \rrbracket_{\Gamma}=0, \quad \llbracket \frac{1}{\rho} \nabla p \rrbracket_{\Gamma} \cdot \boldsymbol{n}_{\Gamma} & =0 & & \text { on } J \times \Gamma, \\
p(0)=p_{0}, \quad \partial_{t} p(0) & =v_{0}, & & \text { in } \Omega, \\
p & =0, & & \text { on } J \times \partial \Omega,
\end{aligned}
$$

where the homogeneous Dirichlet boundary condition (1d) is considered for simplicity. The data are $f \in L^{2}\left(J ; L^{2}(\Omega)\right), p_{0} \in H_{0}^{1}(\Omega)$, and $v_{0} \in H_{0}^{1}(\Omega)$. For simplicity, we assume that the material parameters $\kappa$ and $\rho$ are constant in each subdomain $\Omega_{i}, i \in\{1,2\}$, and we set $\kappa_{i}:=\kappa_{\mid \Omega_{i}}$ and 
$\rho_{i}:=\rho_{\mid \Omega_{i}}$. Recall that $c_{i}:=\left(\frac{\kappa_{i}}{\rho_{i}}\right)^{\frac{1}{2}}$ is the wave speed in each subdomain $\Omega_{i}$. The unit normal vector $\boldsymbol{n}_{\Gamma}$ to $\Gamma$ conventionally points from $\Omega_{1}$ to $\Omega_{2}$ where the numbering of the subdomains corresponds to $\rho_{1} \geq \rho_{2}$. For a smooth enough function $v$ defined on $\Omega_{1} \cup \Omega_{2}$, we denote its jump across $\Gamma$ as $\llbracket v \rrbracket_{\Gamma}:=v_{\mid \Omega_{1}}-v_{\mid \Omega_{2}}$. Notice that we could also consider the more general jump conditions $\llbracket p \rrbracket_{\Gamma}=g_{D} \in L^{2}\left(J ; H^{\frac{1}{2}}(\Gamma)\right)$ and $\llbracket \frac{1}{\rho} \nabla p \rrbracket_{\Gamma} \cdot \boldsymbol{n}_{\Gamma}=g_{N} \in L^{2}\left(J ; L^{2}(\Gamma)\right)$.

\section{Setting for unfitted HHO methods}

In this section, we introduce the notation for unfitted meshes and define the key ingredients for the unfitted HHO method.

\subsection{Unfitted meshes}

Let $\left(\mathcal{T}_{h}\right)_{h>0}$ be a sequence of meshes of $\Omega$. The meshes can have cells that are polyhedra in $\mathbb{R}^{d}$ with planar faces, and hanging nodes are also possible. The mesh cells are taken to be open sets in $\mathbb{R}^{d}$. For all $T \in \mathcal{T}_{h}, h_{T}$ denotes the diameter of the cell $T$ and $\boldsymbol{n}_{T}$ the unit normal on $\partial T$ pointing outward $T$. We set conventionally $h:=\max _{T \in \mathcal{T}_{h}} h_{T}$. The mesh sequence is assumed to be shape-regular, i.e., for all $h>0, \mathcal{T}_{h}$ admits a matching simplicial sub-mesh $\mathcal{T}_{h}^{\prime}$ such that any cell (or face) of $\mathcal{T}_{h}^{\prime}$ is a subset of a cell (or face) of $\mathcal{T}_{h}$, and there exists a mesh-regularity parameter $\rho>0$ such that for all $h>0$, all $T \in \mathcal{T}_{h}$, and all $S \in \mathcal{T}_{h}^{\prime}$ such that $S \subset T$, we have $\rho h_{S} \leq r_{S}$ and $\rho h_{T} \leq h_{S}$, where $r_{S}$ denotes the inradius of the simplex $S$. The mesh faces are collected in the set $\mathcal{F}_{h}$.

Let us define the partition $\mathcal{T}_{h}=\mathcal{T}_{h}^{1} \cup \mathcal{T}_{h}^{\Gamma} \cup \mathcal{T}_{h}^{2}$, where the subsets

$$
\begin{aligned}
\mathcal{T}_{h}^{i} & :=\left\{T \in \mathcal{T}_{h} \mid T \subset \Omega_{i}\right\} \quad \forall i \in\{1,2\}, \\
\mathcal{T}_{h}^{\Gamma} & :=\left\{T \in \mathcal{T}_{h} \mid T \cap \Gamma \neq \emptyset\right\},
\end{aligned}
$$

collect respectively the mesh cells inside the subdomain $\Omega_{i}, i \in\{1,2\}$ (the uncut cells), and those cut by the interface $\Gamma$ (the cut cells). For every cut cell $T \in \mathcal{T}_{h}^{\Gamma}$ and all $i \in\{1,2\}$, we define

$$
T^{i}:=T \cap \Omega_{i}, \quad T^{\Gamma}:=T \cap \Gamma .
$$

For all $i \in\{1,2\}$, the boundary $\partial\left(T^{i}\right)$ of the sub-cell $T^{i}$ is decomposed as

$$
\partial\left(T^{i}\right):=(\partial T)^{i} \cup T^{\Gamma}, \quad(\partial T)^{i}:=\partial T \cap\left(\overline{\Omega_{i}} \backslash \Gamma\right) .
$$

To unify the notation, for every uncut cell $T \in \mathcal{T}_{h}^{i}, i \in\{1,2\}$, we set

$$
T^{i}:=T, \quad T^{\bar{\imath}}:=\emptyset, \quad(\partial T)^{i}:=\partial T, \quad(\partial T)^{\bar{\imath}}:=\emptyset, \quad T^{\Gamma}:=\emptyset,
$$

where $\bar{\imath}:=3-i($ so that $\overline{1}=2$ and $\overline{2}=1)$.

For a subset $S \subset \mathbb{R}^{d}$ consisting of one mesh (sub)cell or one mesh (sub)face, we denote $\mathbb{P}^{\ell}(S)$ (resp. $\left.\mathbb{P}^{\ell}\left(S ; \mathbb{R}^{d}\right)\right)$ the space composed of the restriction to $S$ of scalar-valued (resp. vector-valued) polynomials of total degree at most $\ell \geq 0$. Whenever $S=\emptyset$, we abuse the notation by writing $\mathbb{P}^{\ell}(S):=\{0\}$ and $(\cdot, \cdot)_{L^{2}(S)}:=0$.

Three assumptions on the mesh are needed. The first one quantifies how well the interface cuts the mesh cells, the second one quantifies how well the mesh resolves the interface and the third one requires the mesh not to be excessively graded. In the sequel, $B(\boldsymbol{x}, r)$ denotes the ball of center $\boldsymbol{x}$ and radius $r$. 
Assumption 3.1 (Cut cells). There is $\delta \in(0,1)$ such that, for all $T \in \mathcal{T}_{h}^{\Gamma}$ and all $i \in\{1,2\}$, there is $\tilde{\boldsymbol{x}}_{T^{i}} \in T^{i}$ such that $B\left(\tilde{\boldsymbol{x}}_{T^{i}}, \delta h_{T}\right) \subset T^{i}$.

Assumption 3.2 (Resolving $\Gamma$ ). There is $\gamma \in(0,1)$ such that, for all $T \in \mathcal{T}_{h}^{\Gamma}$, there is a point $\check{\boldsymbol{x}}_{T} \in \mathbb{R}^{d}$ such that setting $T^{\dagger}:=B\left(\check{\boldsymbol{x}}_{T}, \gamma^{-1} h_{T}\right)$ we have the following properties: (i) $T \subset T^{\dagger}$; (ii) for all $\boldsymbol{s} \in T^{\Gamma}, d\left(\check{\boldsymbol{x}}_{T}, T_{\boldsymbol{s}} \Gamma\right) \geq \gamma h_{T}$, where $T_{\boldsymbol{s}} \Gamma$ is the tangent plane to $\Gamma$ at the point $\boldsymbol{s}$; (iii) For all $F \in \mathcal{F}_{\partial T}$, there is $\boldsymbol{x}_{F} \in T^{\dagger}$ such that $d\left(\boldsymbol{x}_{F}, F\right) \geq \gamma h_{T}$.

Assumption 3.3 (Mild mesh grading). For all $T \in \mathcal{T}_{h}$, let the neighboring layers $\Delta_{j}(T) \subset \mathbb{R}^{d}$ be defined by induction as $\Delta_{0}(T):=T$ and $\Delta_{j+1}(T):=\left\{T^{\prime} \in \mathcal{T}_{h} \mid \overline{T^{\prime}} \cap \overline{\Delta_{j}(T)} \neq \emptyset\right\}$ for all $j \in \mathbb{N}$. There is $n_{0} \in \mathbb{N}$ such that for all $T \in \mathcal{T}_{h}$, the ball $T^{\dagger}$ introduced in Assumption 3.2 satisfies $T^{\dagger} \subset \Delta_{n_{0}}(T)$.

It is shown in $[10,6]$ that if the mesh is fine enough, it is possible to devise a cell agglomeration procedure so that, choosing the parameter $\delta$ small enough (depending on the regularity parameter $\rho$ ), Assumption 3.1 is fulfilled. It is shown in [10, Lemma 6.1] that if the mesh is fine enough with respect to the curvature of the interface, the points (i) and (ii) of Assumption 3.2 hold true. Moreover the point (iii) of this assumption can be established by invoking the shape-regularity of the mesh as shown in the proof of [10, Lemma 3.3]. The role of Assumption 3.1 in the analysis is to provide discrete (inverse) inequalities in the cut cells (see [10, Lemma 3.4] and [6, Lemma 3.4 ] for proofs). The role of Assumption 3.2 is to provide a multiplicative trace inequality that is needed to establish optimal approximation properties on the faces and on the interface within the cut cells (see [10, Lemma 3.3] for the proof).

Lemma 3.4 (Discrete inverse inequalities). Let Assumption 3.1 be fulfilled. Let $\ell \in \mathbb{N}$. There is $c_{\mathrm{disc}}$, depending on $\rho, \delta$, and $\ell$, such that, for all $T \in \mathcal{T}_{h}$, all $i \in\{1,2\}$ and all $v_{T^{i}} \in \mathbb{P}^{\ell}\left(T^{i}\right)$, the following inequalities hold true:

- (Discrete trace inequality) $\left\|v_{T^{i}}\right\|_{\partial\left(T^{i}\right)} \leq c_{\mathrm{disc}} h_{T^{-\frac{1}{2}}}^{-1}\left\|v_{T^{i}}\right\|_{T^{i}}$.

- (Discrete inverse inequality) $\left\|\nabla v_{T^{i}}\right\|_{T^{i}} \leq c_{\mathrm{disc}} h_{T}^{-1}\left\|v_{T^{i}}\right\|_{T^{i}}$.

- (Discrete Poincaré inequality) Assuming that $\left(v_{T^{i}}, 1\right)_{B\left(\tilde{\boldsymbol{x}}_{T^{i}}, h_{T}\right)}=0$, we have $\left\|v_{T^{i}}\right\|_{T^{i}} \leq$ $c_{\mathrm{disc}} h_{T}\left\|\nabla v_{T^{i}}\right\|_{T^{i}}$.

Lemma 3.5 (Multiplicative trace inequality). Let Assumption 3.2 be fulfilled. There is $c_{\mathrm{mtr}}>0$, depending on $\rho$ and $\gamma$, such that for all $T \in \mathcal{T}_{h}$, all $v \in H^{1}\left(T^{\dagger}\right)$, and all $i \in\{1,2\}$,

$$
\|v\|_{\partial\left(T^{i}\right)} \leq c_{\mathrm{mtr}}\left(h_{T}^{-\frac{1}{2}}\|v\|_{T^{\dagger}}+\|v\|_{T^{\dagger}}^{\frac{1}{2}}\|\nabla v\|_{T^{\dagger}}^{\frac{1}{2}}\right) .
$$

In what follows, we use the convention $A \lesssim B$ to abbreviate the inequality $A \leq C B$ for positive real numbers $A$ and $B$, where the constant $C$ only depends on the polynomial degree $k \geq 0$, the mesh parameters $\rho, \delta, \gamma$ and $n_{0}$, and the above constants $c_{\mathrm{disc}}$ and $c_{\mathrm{mtr}}$.

\subsection{Discrete unknowns for unfitted HHO methods}

Let $k \geq 0$ be the polynomial degree used in the unfitted HHO method. For every uncut cell $T \in \mathcal{T}_{h}^{i}, i \in\{1,2\}$, the local discrete HHO unknowns are a pair of functions: one polynomial of degree at most $(k+1)$ attached to the cell $T$ and one polynomial of degree at most $k$ attached to each face $F \in \mathcal{F}_{\partial T}$, where $\mathcal{F}_{\partial T}$ comprises all the faces composing the boundary $\partial T$ of the cell $T$. We use the notation

$$
\hat{v}_{T}:=\left(v_{T}, v_{\partial T}\right) \in \mathbb{P}^{k+1}(T) \times \mathbb{P}^{k}\left(\mathcal{F}_{\partial T}\right),
$$


with $\mathbb{P}^{k}\left(\mathcal{F}_{\partial T}\right):=\chi_{F \in \mathcal{F}_{\partial T}} \mathbb{P}^{k}(F)$. For every cut cell $T \in \mathcal{T}_{h}^{\Gamma}$, we double the HHO unknowns, so as to have the usual $\mathrm{HHO}$ unknowns available on each sub-cell, up to the interface $\Gamma$ where there are no unknowns. Thus, the local HHO unknowns in every cut cell $T \in \mathcal{T}_{h}^{\Gamma}$ are

$$
\hat{v}_{T}:=\left(v_{T^{1}}, v_{T^{2}}, v_{(\partial T)^{1}}, v_{(\partial T)^{2}}\right) \in \hat{V}_{T}:=\mathbb{P}^{k+1}\left(T^{1}\right) \times \mathbb{P}^{k+1}\left(T^{2}\right) \times \mathbb{P}^{k}\left(\mathcal{F}_{(\partial T)^{1}}\right) \times \mathbb{P}^{k}\left(\mathcal{F}_{(\partial T)^{2}}\right),
$$

where $\mathcal{F}_{(\partial T)^{i}}:=\left\{F^{i}:=F \cap \Omega_{i} \mid F \in \mathcal{F}_{\partial T}\right\}$ is the collection of the (sub)faces composing $(\partial T)^{i}$ and $\mathbb{P}^{k}\left(\mathcal{F}_{\left.(\partial T)^{i}\right)}\right)=\chi_{F^{i} \in \mathcal{F}_{(\partial T)^{i}}} \mathbb{P}^{k}\left(F^{i}\right), i \in\{1,2\}$. It is also convenient to regroup the local unknowns on each side of the interface by means of the notation $\hat{v}_{T^{i}}:=\left(v_{T^{i}}, v_{(\partial T)^{i}}\right) \in \mathbb{P}^{k+1}\left(T^{i}\right) \times \mathbb{P}^{k}\left(\mathcal{F}_{(\partial T)^{i}}\right)$, $i \in\{1,2\}$. The HHO unknowns are shown in Fig. 1, where the number of bullets represents the number of degrees of freedom attached to each geometric entity. The left panel of Fig. 1 shows three uncut cells, the central panel shows three cut cells, and the right panel shows the result of local agglomeration for the three cut cells. To unify the notation between cut and uncut cells, we define $\hat{V}_{T}$ as in (8) for every uncut cell $T \in \mathcal{T}_{h}^{i}, i \in\{1,2\}$. With this abuse of notation, we have $\hat{v}_{T}:=\left(v_{T}, 0, v_{\partial T}, 0\right)$ for all $T \in \mathcal{T}_{h}^{1}$ and $\hat{v}_{T}:=\left(0, v_{T}, 0, v_{\partial T}\right)$ for all $T \in \mathcal{T}_{h}^{2}$.
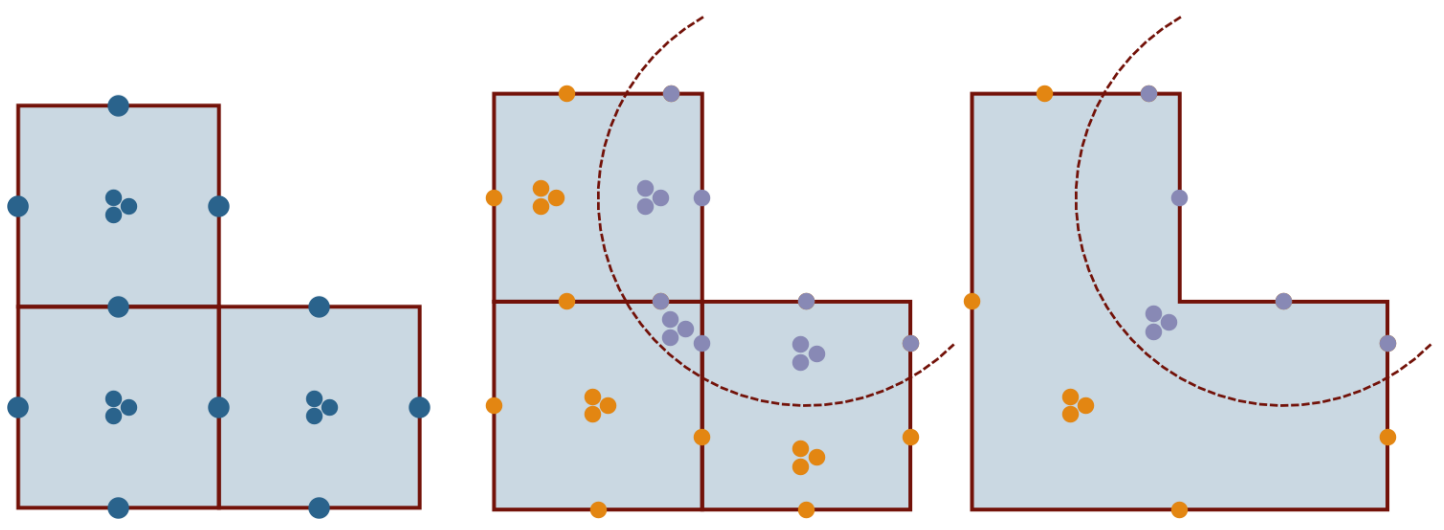

Figure 1: Local HHO unknowns for $k=0$. Left: three uncut cells; center: three cut cells; right: the three cut cells after local agglomeration into one polyhedral cell. Each bullet represents one basis function, and the red dashed line represents the interface $\Gamma$.

\subsection{Local gradient reconstruction}

As usual in HHO methods, a key ingredient is a local gradient reconstruction operator. In every cut cell $T \in \mathcal{T}_{h}^{\Gamma}$, there are two gradient reconstruction operators $\boldsymbol{G}_{T^{i}}^{k}: \hat{V}_{T} \rightarrow \mathbb{P}^{k}\left(T^{i} ; \mathbb{R}^{d}\right)$, $i \in\{1,2\}$ (an alternative choice is to reconstruct the gradient in $\nabla \mathbb{P}^{k+1}\left(T^{i}\right)$ in the spirit of the original HHO method $[18,17]$; the present choice is more suitable in view of extensions to nonlinear problems). For every cut cell $T \in \mathcal{T}_{h}^{\Gamma}$ and every $\hat{v}_{T} \in \hat{V}_{T}$, letting $\llbracket v_{T} \rrbracket_{\Gamma}:=v_{T^{1}}-v_{T^{2}}$, we set

$$
\begin{aligned}
& \left(\boldsymbol{G}_{T^{1}}^{k}\left(\hat{v}_{T}\right), \boldsymbol{q}\right)_{T^{1}}:=\left(\nabla v_{T^{1}}, \boldsymbol{q}\right)_{T^{1}}+\left(v_{(\partial T)^{1}}-v_{T^{1}}, \boldsymbol{q} \cdot \boldsymbol{n}_{T}\right)_{(\partial T)^{1}}-\left(\llbracket v_{T} \rrbracket_{\Gamma}, \boldsymbol{q} \cdot \boldsymbol{n}_{\Gamma}\right)_{T^{\Gamma}}, \\
& \left(\boldsymbol{G}_{T^{2}}^{k}\left(\hat{v}_{T}\right), \boldsymbol{q}\right)_{T^{2}}:=\left(\nabla v_{T^{2}}, \boldsymbol{q}\right)_{T^{2}}+\left(v_{(\partial T)^{2}}-v_{T^{2}}, \boldsymbol{q} \cdot \boldsymbol{n}_{T}\right)_{(\partial T)^{2}},
\end{aligned}
$$

for all $\boldsymbol{q} \in \mathbb{P}^{k}\left(T^{1} ; \mathbb{R}^{d}\right)$ in $(9)$ and all $\boldsymbol{q} \in \mathbb{P}^{k}\left(T^{2} ; \mathbb{R}^{d}\right)$ in (10). Notice that $\boldsymbol{G}_{T^{2}}^{k}\left(\hat{v}_{T}\right)$ only depends on $\hat{v}_{T^{2}}$, whereas $\boldsymbol{G}_{T^{1}}^{k}\left(\hat{v}_{T}\right)$ depends on both $\hat{v}_{T^{1}}$ and $\hat{v}_{T^{2}}$ owing to the jump term on the right-hand side of (9). The difference in the reconstruction between the two subdomains is not essential here 
since we do not explore the strongly contrasted case, and one can also consider the reconstruction discussed in [6, Sec. 2.5]. Using the above conventions on the notation, we use the same definitions for every uncut cell $T \in \mathcal{T}_{h}^{i}, i \in\{1,2\}$, leading to $\left(\boldsymbol{G}_{T^{i}}^{k}\left(\hat{v}_{T}\right), \boldsymbol{q}\right)_{T^{i}}:=\left(\nabla v_{T^{i}}, \boldsymbol{q}\right)_{T^{i}}+\left(v_{(\partial T)^{i}}-\right.$ $\left.v_{T^{i}}, \boldsymbol{q} \cdot \boldsymbol{n}_{T}\right)_{(\partial T)^{i}}$ for all $\boldsymbol{q} \in \mathbb{P}^{k}\left(T^{i} ; \mathbb{R}^{d}\right)$, and $\boldsymbol{G}_{T^{i}}^{k}\left(\hat{v}_{T}\right):=\mathbf{0}$. Recalling that $T^{i}:=T$ and $(\partial T)^{i}:=$ $\partial T, \boldsymbol{G}_{T^{i}}^{k}\left(\hat{v}_{T}\right)$ corresponds to one of the possible HHO gradient reconstructions in the fitted case (see, e.g., $[4,1])$.

\section{Wave equation: second-order formulation}

In this section, we show how to apply the unfitted HHO method to the second-order formulation in time of the acoustic wave equation. Let us set for all $i \in\{1,2\}$,

$$
V_{\mathcal{T}^{i}}:=\underset{T \in \mathcal{T}_{h}}{\chi} \mathbb{P}^{k+1}\left(T^{i}\right), \quad V_{\mathcal{F}^{i}}:=\underset{F \in \mathcal{F}_{h}}{\chi} \mathbb{P}^{k}\left(F^{i}\right)
$$

We define the global discrete spaces

$$
\hat{V}_{h}:=V_{\mathcal{T}} \times V_{\mathcal{F}}, \quad V_{\mathcal{T}}:=V_{\mathcal{T}^{1}} \times V_{\mathcal{T}^{2}}, \quad V_{\mathcal{F}}:=V_{\mathcal{F}^{1}} \times V_{\mathcal{F}^{2}} .
$$

For all $\hat{v}_{h} \in \hat{V}_{h}$, we write $\hat{v}_{h}=\left(v_{\mathcal{T}}, v_{\mathcal{F}}\right)$ with $v_{\mathcal{T}}=\left(v_{\mathcal{T}^{1}}, v_{\mathcal{T}^{2}}\right) \in V_{\mathcal{T}}$ and $v_{\mathcal{F}}=\left(v_{\mathcal{F}^{1}}, v_{\mathcal{F}^{2}}\right) \in V_{\mathcal{F}}$. Moreover, for all $T$ in $\mathcal{T}_{h}$, we denote $\hat{v}_{T}:=\left(v_{T^{1}}, v_{T^{2}}, v_{(\partial T)^{1}}, v_{(\partial T)^{2}}\right)$ the local components of $\hat{v}_{h}$ respectively attached to $T^{1}, T^{2},(\partial T)^{1}$, and $(\partial T)^{2}$. We denote $\hat{V}_{h 0}$ the subspace of $\hat{V}_{h}$ where all degrees of freedom attached to the faces composing $\partial \Omega$ are null.

The global discrete bilinear form is assembled cellwise by summing the contributions of local discrete bilinear forms. For all $T \in \mathcal{T}_{h}$ and all $\hat{v}_{T}, \hat{w}_{T} \in \hat{V}_{T}$, we set

$$
b_{T}\left(\hat{v}_{T}, \hat{w}_{T}\right):=\sum_{i \in\{1,2\}}\left\{\left(\boldsymbol{G}_{T^{i}}^{k}\left(\hat{v}_{T}\right), \boldsymbol{G}_{T^{i}}^{k}\left(\hat{w}_{T}\right)\right)_{\rho_{i}^{-1} ; T^{i}}+s_{T^{i}}\left(\hat{v}_{T^{i}}, \hat{w}_{T^{i}}\right)\right\}+s_{T}^{\Gamma}\left(\hat{v}_{T}, \hat{w}_{T}\right),
$$

with

$$
\begin{aligned}
s_{T^{i}}\left(\hat{v}_{T^{i}}, \hat{w}_{T^{i}}\right) & :=\left(\rho_{i} h_{T}\right)^{-1}\left(\Pi_{(\partial T)^{i}}^{k}\left(v_{T^{i}}-v_{(\partial T)^{i}}\right), w_{T^{i}}-w_{(\partial T)^{i}}\right)_{(\partial T)^{i}}, \\
s_{T}^{\Gamma}\left(\hat{v}_{T}, \hat{w}_{T}\right) & :=\left(\rho_{1} h_{T}\right)^{-1}\left(\llbracket v_{T} \rrbracket_{\Gamma}, \llbracket w_{T} \rrbracket_{\Gamma}\right)_{T^{\Gamma}},
\end{aligned}
$$

where $\Pi_{(\partial T)^{i}}^{k}$ denotes the $L^{2}$-orthogonal projector onto $\mathbb{P}^{k}\left(\mathcal{F}_{(\partial T)^{i}}\right)$. Notice that the role of $s_{T^{i}}$ is to weakly enforce the matching between cell- and face-based HHO unknowns in all the faces inside the subdomains, whereas the role of $s_{T}^{\Gamma}$ is to weakly enforce the first jump condition in (1b). We next define the global bilinear form $b_{h}: \hat{V}_{h} \times \hat{V}_{h} \rightarrow \mathbb{R}$ such that

$$
b_{h}\left(\hat{v}_{h}, \hat{w}_{h}\right):=\sum_{T \in \mathcal{T}_{h}} b_{T}\left(\hat{v}_{T}, \hat{w}_{T}\right)=\left(\boldsymbol{G}_{\mathcal{T}}^{k}\left(\hat{v}_{h}\right), \boldsymbol{G}_{\mathcal{T}}^{k}\left(\hat{w}_{h}\right)\right)_{\rho^{-1} ; \Omega}+s_{h}\left(\hat{v}_{h}, \hat{w}_{h}\right)+s_{h}^{\Gamma}\left(\hat{v}_{h}, \hat{w}_{h}\right)
$$

with the global reconstruction operator such that $\boldsymbol{G}_{\mathcal{T}}^{k}\left(\hat{v}_{h}\right)_{\mid T^{i}}:=\boldsymbol{G}_{T^{i}}\left(\hat{v}_{T}\right)$ for all $T \in \mathcal{T}_{h}$, all $i \in\{1,2\}$, and all $\hat{v}_{h} \in \hat{V}_{h}$, and the global stabilization bilinear forms such that $s_{h}\left(\hat{v}_{h}, \hat{w}_{h}\right):=$ $\sum_{T \in \mathcal{T}_{h}} \sum_{i \in\{1,2\}} s_{T^{i}}\left(\hat{v}_{T^{i}}, \hat{w}_{T^{i}}\right)$ and $s_{h}^{\Gamma}\left(\hat{v}_{h}, \hat{w}_{h}\right):=\sum_{T \in \mathcal{T}_{h}} s_{T}^{\Gamma}\left(\hat{v}_{T}, \hat{w}_{T}\right)$.

The space semi-discrete HHO scheme for the second-order wave equation consists of finding $\hat{p}_{h}:=\left(p_{\mathcal{T}}, p_{\mathcal{F}}\right) \in C^{2}\left(\bar{J} ; \hat{V}_{h 0}\right)$ such that for all $t \in \bar{J}$,

$$
\left(\partial_{t t} p_{\mathcal{T}}(t), q_{\mathcal{T}}\right)_{\frac{1}{\kappa} ; \Omega}+b_{h}\left(\hat{p}_{h}(t), \hat{q}_{h}\right)=\left(f(t), q_{\mathcal{T}}\right)_{\Omega}
$$


for all $\hat{q}_{h}:=\left(q_{\mathcal{T}}, q_{\mathcal{F}}\right) \in \hat{V}_{h 0}$. The initial conditions for (17) only concern $p_{\mathcal{T}}$ and are as follows:

$$
p_{\mathcal{T}}(0)_{\mid \Omega_{i}}=\Pi_{\mathcal{T}^{i}}^{k+1}\left(p_{0 \mid \Omega_{i}}\right), \quad \partial_{t} p_{\mathcal{T}}(0)_{\mid \Omega_{i}}=\Pi_{\mathcal{T}^{i}}^{k+1}\left(v_{0 \mid \Omega_{i}}\right), \quad i \in\{1,2\},
$$

where $\Pi_{\mathcal{T}^{i}}^{k+1}$ is the $L^{2}$-orthogonal projection onto $V_{\mathcal{T}^{i}}, i \in\{1,2\}$. The boundary condition is encoded in the fact that $\hat{p}_{h}(t) \in \hat{V}_{h 0}$ for all $t \in \bar{J}$. Notice that since the space semi-discrete solution is smooth in time, (17) holds at the initial time which implies that $p_{\mathcal{F}}(0) \in V_{\mathcal{F}}$ is uniquely determined by the equations $b_{h}\left(\left(p_{\mathcal{T}}(0), p_{\mathcal{F}}(0)\right),\left(0, q_{\mathcal{F}}\right)\right)=0$ for all $q_{\mathcal{F}} \in V_{\mathcal{F}}$ with $p_{\mathcal{T}}(0)$ specified in (18) and the boundary condition enforcing to zero the components of $p_{\mathcal{F}}(0)$ attached to the boundary faces.

Let $N_{\mathcal{T}}:=\operatorname{dim}\left(V_{\mathcal{T}}\right)$ and $N_{\mathcal{F}}:=\operatorname{dim}\left(V_{\mathcal{F}}\right)$. Let $\left(\mathrm{P}_{\mathcal{T}}(t), \mathrm{P}_{\mathcal{F}}(t)\right) \in \mathbb{R}^{N_{\mathcal{T}} \times N_{\mathcal{F}}}$ be the (timedependent) component vectors of the space semi-discrete solution $\hat{p}_{h}(t):=\left(p_{\mathcal{T}}(t), p_{\mathcal{F}}(t)\right) \in \hat{V}_{h 0}$ once bases $\left\{\varphi_{i}\right\}_{1 \leq i \leq N_{\mathcal{T}}}$ and $\left\{\psi_{j}\right\}_{1 \leq j \leq N_{\mathcal{F}}}$ for $V_{\mathcal{T}}$ and $V_{\mathcal{F}}$, respectively, have been chosen. Let $\mathrm{F}_{\mathcal{T}}(t) \in \mathbb{R}^{N_{\mathcal{T}}}$ have components given by $\mathrm{F}_{i}(t):=\left(f(t), \varphi_{i}\right)_{\Omega}$ for all $t \in \bar{J}$ and all $1 \leq i \leq N_{\mathcal{T}}$. The algebraic realization of (18) is as follows: For all $t \in \bar{J}$,

$$
\left[\begin{array}{cc}
\mathrm{M}_{\mathcal{T} \mathcal{T}} & 0 \\
0 & 0
\end{array}\right]\left[\begin{array}{c}
\partial_{t t} \mathrm{P}_{\mathcal{T}}(t) \\
\bullet
\end{array}\right]+\left[\begin{array}{ll}
\mathrm{K}_{\mathcal{T} \mathcal{T}} & \mathrm{K}_{\mathcal{T} \mathcal{F}} \\
\mathrm{K}_{\mathcal{F} \mathcal{T}} & \mathrm{K}_{\mathcal{F} \mathcal{F}}
\end{array}\right]\left[\begin{array}{l}
\mathrm{P}_{\mathcal{T}}(t) \\
\mathrm{P}_{\mathcal{F}}(t)
\end{array}\right]=\left[\begin{array}{c}
\mathrm{F}_{\mathcal{T}}(t) \\
0
\end{array}\right]
$$

with the mass matrix $\mathbf{M}_{\mathcal{T} \mathcal{T}}$ associated with the inner product in $L^{2}\left(\frac{1}{\kappa} ; \Omega\right)$ and the cell basis functions, and the symmetric positive-definite stiffness matrix with blocks $\mathrm{K}_{\mathcal{T} \mathcal{T}}, \mathrm{K}_{\mathcal{T} \mathcal{F}}, \mathrm{K}_{\mathcal{F} \mathcal{T}}$, $\mathrm{K}_{\mathcal{F F}}$ associated with the bilinear form $b_{h}$ and the cell and face basis functions. The bullet stands for $\partial_{t t} \mathrm{P}_{\mathcal{F}}(t)$ which is irrelevant owing to the structure of the mass matrix. The matrices $\mathbf{M}_{\mathcal{T} \mathcal{T}}$ and $\mathrm{K}_{\mathcal{T} \mathcal{T}}$ are block-diagonal, but not the matrix $\mathrm{K}_{\mathcal{F F}}$ since the components attached to faces belonging to the same cell are coupled together. Notice also that the sizes of the diagonal blocks $\mathrm{M}_{\mathcal{T} \mathcal{T}}$ and $\mathrm{K}_{\mathcal{T} \mathcal{T}}$ are different for the uncut and the cut cells.

Remark 4.1 (Fitted case). In the fitted case, the above HHO method has been devised in [8]. Moreover, using the ideas from [13] to bridge HHO and HDG methods, this method can be connected to the space semi-discrete HDG formulation from [14]. Notice that unfitted HHO methods use a mixed-order polynomial setting where the polynomials attached to the cells are one order higher than those attached to the faces. The stabilization bilinear form (14) on the faces inside each subdomain amounts to the Lehrenfeld-Schöberl HDG stabilization [26, 27].

\section{Wave equation: first-order formulation}

In this section, we apply the unfitted HHO method to the first-order formulation in time of the acoustic wave equation. The first-order formulation is classically obtained by introducing two auxiliary variables, the scalar velocity $v:=\partial_{t} p$ and the dual variable $\sigma:=\frac{1}{\rho} \nabla p$. This leads to the following coupled PDEs:

$$
\left\{\begin{aligned}
\rho \partial_{t} \boldsymbol{\sigma}-\nabla v & =\mathbf{0} \\
\frac{1}{\kappa} \partial_{t} v-\nabla \cdot \boldsymbol{\sigma} & =f
\end{aligned} \quad \text { in } J \times\left(\Omega_{1} \cup \Omega_{2}\right),\right.
$$

together with the boundary condition $v=0$ on $J \times \Gamma$ and the initial conditions $v(0)=v_{0}$ and $\boldsymbol{\sigma}(0)=\frac{1}{\rho} \nabla p_{0}$ in $\Omega$. The jump conditions become $\llbracket v \rrbracket_{\Gamma}=0$ and $\llbracket \boldsymbol{\sigma} \rrbracket_{\Gamma} \cdot \boldsymbol{n}_{F}=0$ on $\Gamma$.

In the space semi-discrete case, one approximates $v$ by a hybrid unknown $\hat{v}_{h} \in C^{1}\left(\bar{J} ; \hat{V}_{h 0}\right)$ and $\boldsymbol{\sigma}$ by a cellwise unknown $\boldsymbol{\sigma}_{\mathcal{T}} \in C^{1}\left(\bar{J} ; \boldsymbol{W}_{\mathcal{T}}\right)$ where

$$
\boldsymbol{W}_{\mathcal{T}}:=\boldsymbol{W}_{\mathcal{T}^{1}} \times \boldsymbol{W}_{\mathcal{T}^{2}}, \quad \boldsymbol{W}_{\mathcal{T}^{i}}:=\underset{T \in \mathcal{T}_{h}}{\chi} \mathbb{P}^{k}\left(T^{i} ; \mathbb{R}^{d}\right) .
$$


The space semi-discrete problem reads as follows: For all $t \in \bar{J}$,

$$
\left\{\begin{aligned}
\left(\partial_{t} \boldsymbol{\sigma}_{\mathcal{T}}(t), \boldsymbol{\tau}_{\mathcal{T}}\right)_{\rho ; \Omega}-\left(\boldsymbol{G}_{\mathcal{T}}\left(\hat{v}_{h}(t)\right), \boldsymbol{\tau}_{\mathcal{T}}\right)_{\Omega} & =0 \\
\left(\partial_{t} v_{\mathcal{T}}(t), w_{\mathcal{T}}\right)_{\frac{1}{\kappa} ; \Omega}+\left(\boldsymbol{\sigma}_{\mathcal{T}}(t), \boldsymbol{G}_{\mathcal{T}}\left(\hat{w}_{h}\right)\right)_{\boldsymbol{L}^{2}(\Omega)}+\tilde{s}_{h}\left(\hat{v}_{h}(t), \hat{w}_{h}\right)+\tilde{s}_{h}^{\Gamma}\left(\hat{v}_{h}(t), \hat{w}_{h}\right) & =\left(f(t), w_{\mathcal{T}}\right)_{\Omega},
\end{aligned}\right.
$$

for all $\left(\boldsymbol{\tau}_{\mathcal{T}}, \hat{w}_{h}\right) \in \boldsymbol{W}_{\mathcal{T}} \times \hat{V}_{h 0}$, together with the initial conditions $\boldsymbol{\sigma}_{\mathcal{T}}(0)=\frac{1}{\rho} \boldsymbol{G}_{\mathcal{T}}\left(\Pi_{\mathcal{T}}^{k+1}\left(p_{0}\right), \Pi_{\mathcal{F}}^{k}\left(p_{0}\right)\right)$ and $v(0)=\Pi_{\mathcal{T}}^{k+1}\left(v_{0}\right)$, where $\boldsymbol{G}_{\mathcal{T}}$ is defined in Section $4, \Pi_{\mathcal{T}}^{k+1}:=\left(\Pi_{\mathcal{T}^{1}}^{k+1}, \Pi_{\mathcal{T}^{2}}^{k+1}\right), \Pi_{\mathcal{F}}^{k}:=\left(\Pi_{\mathcal{F}^{1}}^{k}, \Pi_{\mathcal{F}^{2}}^{k}\right)$, $\Pi_{\mathcal{T}^{i}}^{k+1}$ is the $L^{2}$-orthogonal projection onto $V_{\mathcal{T}^{i}}$, and $\Pi_{\mathcal{F}^{i}}^{k}$ that onto $V_{\mathcal{F}^{i}}, i \in\{1,2\}$. Moreover, the stabilization bilinear forms are $\tilde{s}_{h}\left(\hat{v}_{h}, \hat{w}_{h}\right):=\sum_{T \in \mathcal{T}_{h}} \tilde{s}_{T}\left(\hat{v}_{T}, \hat{w}_{T}\right)$ and $\tilde{s}_{h}^{\Gamma}\left(\hat{v}_{h}, \hat{w}_{h}\right):=$ $\sum_{T \in \mathcal{T}_{h}} \tilde{s}_{T}^{\Gamma}\left(\hat{v}_{T}, \hat{w}_{T}\right)$, with (compare with (14)-(15))

$$
\begin{aligned}
& \tilde{s}_{T}\left(\hat{v}_{T}, \hat{w}_{T}\right):=\sum_{i \in\{1,2\}} \tilde{\tau}_{(\partial T)^{i}}\left(\Pi_{(\partial T)^{i}}^{k}\left(v_{T^{i}}-v_{(\partial T)^{i}}\right), w_{T^{i}}-w_{(\partial T)^{i}}\right)_{(\partial T)^{i}}, \\
& \tilde{s}_{T}^{\Gamma}\left(\hat{v}_{T}, \hat{w}_{T}\right):=\tilde{\tau}_{T^{\Gamma}}\left(\llbracket v_{T} \rrbracket_{\Gamma}, \llbracket w_{T} \rrbracket_{\Gamma}\right)_{T^{\Gamma}} .
\end{aligned}
$$

We consider two possible values for the stabilization weights. When using implicit time-stepping schemes, we set $\tilde{\tau}_{(\partial T)^{i}}:=\frac{\ell_{\Omega}}{c_{i}}\left(\rho_{i} h_{T}\right)^{-1}$ and $\tilde{\tau}_{T^{\Gamma}}:=\frac{\ell_{\Omega}}{c_{1}}\left(\rho_{1} h_{T}\right)^{-1}$ (in short, $\tilde{\tau}_{\partial T}=\mathcal{O}\left(h^{-1}\right)$ ), where $\ell_{\Omega}:=\operatorname{diam}(\Omega)$ is a global length scale introduced to remain dimensionally consistent. Instead, when using explicit time-stepping schemes, we set $\tilde{\tau}_{(\partial T)^{i}}:=\left(\rho_{i} c_{i}\right)^{-1}$ and $\tilde{\tau}_{T^{\Gamma}}:=\left(\rho_{1} c_{1}\right)^{-1}$ (in short, $\left.\tilde{\tau}_{\partial T}=\mathcal{O}(1)\right)$. These choices are motivated as follows. For implicit schemes, $\tilde{\tau}_{\partial T}=\mathcal{O}\left(h^{-1}\right)$ has been observed in [8] to produce more accurate solutions than $\tilde{\tau}_{\partial T}=\mathcal{O}(1)$. For explicit schemes, the discussion in Section 7.4 will show that $\tilde{\tau}_{\partial T}=\mathcal{O}\left(h^{-1}\right)$ leads to an unfavorable quadratic scaling with respect to $h$ in the CFL time-step restriction, whereas $\tilde{\tau}_{\partial T}=\mathcal{O}(1)$ leads to the expected first-order scaling.

Let $M_{\mathcal{T}}:=\operatorname{dim}\left(\boldsymbol{W}_{\mathcal{T}}\right)=d N_{\mathcal{T}}^{k}$ and $\left\{\boldsymbol{\zeta}_{i}\right\}_{1 \leq i \leq M_{\mathcal{T}}}$ be the chosen basis for $\boldsymbol{W}_{\mathcal{T}}$. Let $\boldsymbol{Z}_{\mathcal{T}}(t) \in \mathbb{R}^{M_{\mathcal{T}}}$ and $\left(\bigvee_{\mathcal{T}}(t), \bigvee_{\mathcal{F}}(t)\right) \in \mathbb{R}^{N_{\mathcal{T}} \times N_{\mathcal{F}}}$ be the component vectors of $\boldsymbol{\sigma}_{\mathcal{T}}(t) \in \boldsymbol{W}_{\mathcal{T}}$ and $\hat{v}_{h}(t) \in \hat{V}_{h 0}$, respectively. Let $\mathrm{M}_{\mathcal{T} \mathcal{T}}^{\sigma}$ be the mass matrix associated with the inner product in $\boldsymbol{L}^{2}(\rho ; \Omega)$ and the basis functions $\left\{\boldsymbol{\zeta}_{i}\right\}_{1 \leq i \leq M_{\mathcal{T}}}$, and recall that the mass matrix $\mathbf{M}_{\mathcal{T} \mathcal{T}}$ and the right-hand side vector $\mathrm{F}(t)$ are defined in Section 4. Let $\mathrm{S}_{\mathcal{T} \mathcal{T}}, \mathbf{S}_{\mathcal{T F}}, \mathbf{S}_{\mathcal{F} \mathcal{T}}, \mathbf{S}_{\mathcal{F F}}$ be the four blocks composing the matrix representing the stabilization bilinear form $\tilde{s}_{h}+\tilde{s}_{h}^{\Gamma}\left(\tilde{s}_{h}^{\Gamma}\right.$ only contributes to the block $\mathrm{S}_{\mathcal{T} \mathcal{T}}$ ). Let $\mathrm{G}_{\mathcal{T}} \in \mathbb{R}^{M_{\mathcal{T}} \times N_{\mathcal{T}}}$ and $\mathrm{G}_{\mathcal{F}} \in \mathbb{R}^{M_{\mathcal{T} \times N_{\mathcal{F}}}}$ be the (rectangular) matrices representing the gradient reconstruction operator. The algebraic realization of (22) is as follows: For all $t \in \bar{J}$,

$$
\left[\begin{array}{ccc}
\mathrm{M}_{\mathcal{T} \mathcal{T}}^{\boldsymbol{\sigma}} & 0 & 0 \\
0 & \mathrm{M}_{\mathcal{T} \mathcal{T}} & 0 \\
0 & 0 & 0
\end{array}\right]\left[\begin{array}{c}
\partial_{t} \mathrm{Z}_{\mathcal{T}}(t) \\
\partial_{t} \mathrm{~V}_{\mathcal{T}}(t) \\
\bullet
\end{array}\right]+\left[\begin{array}{ccc}
0 & -\mathrm{G}_{\mathcal{T}} & -\mathrm{G}_{\mathcal{F}} \\
\mathrm{G}_{\mathcal{T}}^{\dagger} & \mathrm{S}_{\mathcal{T} \mathcal{T}} & \mathrm{S}_{\mathcal{T} \mathcal{F}} \\
\mathrm{G}_{\mathcal{F}}^{\dagger} & \mathrm{S}_{\mathcal{F} \mathcal{T}} & \mathrm{S}_{\mathcal{F F}}
\end{array}\right]\left[\begin{array}{c}
\mathrm{Z}_{\mathcal{T}}(t) \\
\mathrm{V}_{\mathcal{T}}(t) \\
\mathrm{V}_{\mathcal{F}}(t)
\end{array}\right]=\left[\begin{array}{c}
0 \\
\mathrm{~F}_{\mathcal{T}}(t) \\
0
\end{array}\right]
$$

where the bullet stands for $\partial_{t} \vee_{\mathcal{F}}(t)$ which is irrelevant owing to the structure of the mass matrix. Notice that the third equation in (25) implies that

$$
\mathrm{S}_{\mathcal{F} \mathcal{F}} \mathrm{V}_{\mathcal{F}}(t)=-\left(\mathrm{G}_{\mathcal{F}}^{\dagger} \mathrm{Z}_{\mathcal{T}}(t)+\mathrm{S}_{\mathcal{F} \mathcal{T}} \mathrm{V}_{\mathcal{T}}(t)\right)
$$

and that the sub-matrix $\mathrm{S}_{\mathcal{F} \mathcal{F}}$ is symmetric positive-definite. A crucial observation is that this sub-matrix is additionally block-diagonal, so that (26) is very cheap to invert.

Remark 5.1 (Fitted case). In the fitted case, the above HHO method has been devised in [8]. Owing to [13], this method can be bridged to the space semi-discrete HDG formulation from [29]. Notice though that [29] considers an equal-order setting for the cell and face unknowns and a stability weight $\tilde{\tau}_{\partial T}=\mathcal{O}(1)$. 


\section{Error analysis for the second-order in time formulation}

This section is devoted to the error analysis of the unfitted HHO method for the second-order formulation in time of the acoustic wave equation. The analysis combines ideas from $[10,6]$ concerning the stability and consistency of unfitted HHO methods with ideas from [9] concerning the analysis of fitted HHO methods for the wave equation. These latter ideas in turn draw on the error analysis from [20,3] for continuous finite elements applied to the wave equation. We assume that the assumptions stated in Section 3.1 hold true.

\subsection{Stability, approximation, and consistency}

We equip the space $\hat{V}_{h 0}$ with the norm

$$
\left\|\hat{v}_{h}\right\|_{\hat{V}_{h 0}}^{2}:=\sum_{T \in \mathcal{T}_{h}} \sum_{i \in\{1,2\}}\left\{\left\|\nabla v_{T^{i}}\right\|_{\rho_{i}^{-1} ; T^{i}}^{2}+\left(\rho_{i} h_{T}\right)^{-1}\left\|v_{(\partial T)^{i}}-v_{T^{i}}\right\|_{(\partial T)^{i}}^{2}\right\}+\left(\rho_{1} h_{T}\right)^{-1}\left\|\llbracket v_{T} \rrbracket_{\Gamma}\right\|_{T^{\Gamma}}^{2} .
$$

We have the following important stability result $[6$, Lem. 3.6] (which only requires Assumption 3.1): There are $0<\alpha \leq \omega<+\infty$ such that

$$
\alpha\left\|\hat{v}_{h}\right\|_{\hat{V}_{h 0}}^{2} \leq b_{h}\left(\hat{v}_{h}, \hat{v}_{h}\right) \leq \omega\left\|\hat{v}_{h}\right\|_{\hat{V}_{h 0}}^{2}, \quad \forall \hat{v}_{h} \in \hat{V}_{h 0}
$$

The error analysis hinges on a suitable error decomposition defined by some approximation of the exact solution. To this purpose, following [6], we assume that there is a real number $\nu \in\left(\frac{1}{2}, k+1\right]$ such that the exact solution satisfies $p_{\mid \Omega_{i}} \in C^{1}\left(\bar{J} ; H^{1+\nu}\left(\Omega_{i}\right)\right), i \in\{1,2\}$. The regularity properties of the solution to the homogeneous wave equation are well understood (see, e.g., [21, Sec. 7.2.3]). In contrast, fewer results are available in the heterogeneous case. For two infinite media separated by a hyperplane, regularity results are studied in [36] (see, e.g., Theorem 2.7.3 therein), but we are not aware of regularity results established for more general settings as the one considered herein. Assuming that the above regularity result nonetheless holds true, our analysis then invokes stable extension operators $E_{i}: H^{1+\nu}\left(\Omega_{i}\right) \rightarrow H^{1+\nu}\left(\mathbb{R}^{d}\right)$. For all $v \in H^{1}\left(\Omega_{1} \cup \Omega_{2}\right)$ with $v_{i}:=v_{\mid \Omega_{i}}$ and all $T \in \mathcal{T}_{h}$, we then define

$$
\begin{aligned}
& I_{T^{i}}^{k+1}\left(v_{i}\right):=\left(\Pi_{T^{\dagger}}^{k+1}\left(E_{i}\left(v_{i}\right)_{\mid T^{\dagger}}\right)\right)_{\mid T^{i}} \in \mathbb{P}^{k+1}\left(T^{i}\right), \\
& \hat{I}_{T}^{k}(v):=\left(I_{T^{1}}^{k+1}\left(v_{1}\right), I_{T^{2}}^{k+1}\left(v_{2}\right), \Pi_{(\partial T)^{1}}^{k}\left(v_{1}\right), \Pi_{(\partial T)^{2}}^{k}\left(v_{2}\right)\right) \in \hat{V}_{T},
\end{aligned}
$$

where $\Pi_{T^{\dagger}}^{k+1}$ denotes the $L^{2}$-orthogonal projector onto $\mathbb{P}^{k+1}\left(T^{\dagger}\right)$. Note that we do not project using the sub-cell $T^{i}$ but the larger set $T^{\dagger}$ from Assumption 3.2 so as to invoke the optimal approximation properties of $I_{T^{i}}^{k+1}$ (see for instance [10, Lemma 5.6]). Indeed, if this assumption is fulfilled, we have for all $v \in L^{2}(\Omega)$ such that $v_{i} \in H^{1+\nu}\left(\Omega_{i}\right), i \in\{1,2\}$, and all $T \in \mathcal{T}_{h}$,

$$
\begin{aligned}
& \left\|v_{i}-I_{T^{i}}^{k+1}\left(v_{i}\right)\right\|_{T^{i}}+h_{T}^{\frac{1}{2}}\left\|v_{i}-I_{T^{i}}^{k+1}\left(v_{i}\right)\right\|_{(\partial T)^{i}}+h_{T}\left\|\nabla\left(v_{i}-I_{T^{i}}^{k+1}\left(v_{i}\right)\right)\right\|_{T^{i}} \\
& \lesssim h_{T}^{1+\nu}\left|E_{i}\left(v_{i}\right)\right|_{H^{1+\nu}\left(T^{\dagger}\right)}, \\
& h_{T}^{\frac{1}{2}}\left\|\llbracket v-I_{T}^{k+1}(v) \rrbracket_{\Gamma}\right\|_{T^{\Gamma}} \lesssim h_{T}^{1+\nu} \sum_{j \in\{1,2\}}\left|E_{j}\left(v_{j}\right)\right|_{H^{1+\nu}\left(T^{\dagger}\right)} .
\end{aligned}
$$

The global operator $\hat{I}_{h}^{k}$ is built locally by using the operators $\hat{I}_{T}^{k}$ defined in (30), and the cell component of $\hat{I}_{h}^{k}$ is denoted $I_{\mathcal{T}}^{k+1}$ with its restrictions to each subdomain denoted $I_{\mathcal{T}^{i}}^{k+1}, i \in\{1,2\}$. 
Let $v \in H^{1}(\Omega)$ be such that $v_{i} \in H^{1+\nu}\left(\Omega_{i}\right), i \in\{1,2\}$, and $B(v):=-\nabla \cdot\left(\frac{1}{\rho} \nabla v\right) \in L^{2}(\Omega)$. The consistency of the discrete bilinear form $b_{h}$ is measured by the linear form $\delta_{h}(v ; \cdot) \in\left(\hat{V}_{h 0}\right)^{\prime}$ such that

$$
\delta_{h}\left(v ; \hat{q}_{h}\right):=\left(B(v), q_{\mathcal{T}}\right)_{\Omega}-b_{h}\left(\hat{I}_{h}^{k}(v), \hat{q}_{h}\right), \quad \forall \hat{q}_{h} \in \hat{V}_{h 0} .
$$

Then it is shown in [6, Lem. 3.9] that

$$
\left\|\delta_{h}(v ; \cdot)\right\|_{\left(\hat{V}_{h 0}\right)^{\prime}}:=\sup _{\hat{q}_{h} \in \hat{V}_{h 0} \backslash\{0\}} \frac{\left|\delta_{h}\left(v ; \hat{q}_{h}\right)\right|}{\left\|\hat{q}_{h}\right\|_{\hat{V}_{h 0}}} \lesssim|v|_{*, h},
$$

with

$$
\begin{aligned}
|v|_{*, h}^{2}:= & \sum_{T \in \mathcal{T}_{h}} \sum_{i \in\{1,2\}}\left\{\rho_{i}^{-1}\left(\|\gamma(v)\|_{T^{i}}^{2}+h_{T}\|\gamma(v)\|_{(\partial T)^{i}}^{2}\right)\right\}+\rho_{1}^{-1} h_{T}\|\gamma(v)\|_{T^{\Gamma}}^{2} \\
& +\sum_{T \in \mathcal{T}_{h}} \sum_{i \in\{1,2\}}\left(\rho_{i} h_{T}\right)^{-1}\|\eta(v)\|_{(\partial T)^{i}}^{2}+\left(\rho_{1} h_{T}\right)^{-1}\left\|\llbracket \eta(v) \rrbracket_{\Gamma}\right\|_{T^{\Gamma}}^{2},
\end{aligned}
$$

and $\gamma(v):=\nabla v-\boldsymbol{G}_{\mathcal{T}}\left(\hat{I}_{h}^{k}(v)\right)$ and $\eta(v)_{\mid \Omega_{i}}:=v_{i}-I_{\mathcal{T}^{i}}^{k+1}(v), i \in\{1,2\}$.

\section{2 $\quad H^{1}$-error estimate}

We define the discrete error such that, for all $t \in \bar{J}$,

$$
\hat{e}_{h}(t):=\hat{p}_{h}(t)-\hat{I}_{h}^{k}(p(t)) .
$$

For a function $\hat{v}_{h} \in C^{0}\left(\bar{J} ; \hat{V}_{h 0}\right)$, we set $\left\|\hat{v}_{h}\right\|_{C^{0}\left(0, t ; \hat{V}_{h 0}\right)}:=\sup _{s \in[0, t]}\left\|\hat{v}_{h}(s)\right\|_{\hat{V}_{h 0}}$ for all $t \in J$. We also define $\Omega^{\Gamma}:=\bigcup_{T \in \mathcal{T}_{h}^{\Gamma}} T, \rho_{b}:=\min \left(\rho_{1}, \rho_{2}\right)=\rho_{2}, \rho_{\sharp}:=\max \left(\rho_{1}, \rho_{2}\right)=\rho_{1}, \kappa_{b}:=\min \left(\kappa_{1}, \kappa_{2}\right)$, and $\chi:=\left(\kappa_{b} / \rho_{\sharp}\right)^{\frac{1}{2}}$.

Theorem 6.1 ( $H^{1}$-error estimate). Let $p$ solve (1) and let $\hat{p}_{h}$ solve (17) with the slightly modified initial conditions $p_{\mathcal{T}}(0)_{\mid \Omega_{i}}=I_{\mathcal{T}^{i}}^{k+1}\left(p_{0 \mid \Omega_{i}}\right)$ and $\partial_{t} p_{\mathcal{T}}(0)_{\mid \Omega_{i}}=\Pi_{\mathcal{T}^{i}}^{k+1}\left(v_{0 \mid \Omega_{i}}\right), i \in\{1,2\}$. Assume that $p_{\mid \Omega_{i}} \in C^{3}\left(\bar{J} ; H^{1+\nu}\left(\Omega_{i}\right)\right), i \in\{1,2\}$, with $\nu \in\left(\frac{1}{2}, k+1\right]$. The following holds for all $t \in J$,

$$
\begin{aligned}
\left\|\partial_{t} p_{\mathcal{T}}-I_{\mathcal{T}}^{k+1}\left(\partial_{t} p\right)\right\|_{C^{0}\left(0, t ; L^{2}\left(\frac{1}{\kappa} ; \Omega\right)\right)}+\left\|\hat{p}_{h}-\hat{I}_{h}(p)\right\|_{C^{0}\left(0, t ; \hat{V}_{h 0}\right)} & \\
& \lesssim\left(|p|_{C^{0}(0, t ; *, h)}+\left|\partial_{t} p\right|_{L^{1}(0, t ; *, h)}\right)
\end{aligned}
$$

where we have set

$$
\begin{aligned}
|p|_{C^{0}(0, t ; *, h)} & :=\sup _{s \in[0, t]}\left(|p(s)|_{*, h}+\chi^{-1} \ell_{\Omega}\left\|\partial_{t t} p(s)-I_{\mathcal{T}}^{k+1}\left(\partial_{t t} p(s)\right)\right\|_{\frac{1}{\kappa} ; \Omega^{\Gamma}}\right), \\
\left|\partial_{t} p\right|_{L^{1}(0, t ; *, h)} & :=\int_{0}^{t}\left(\left|\partial_{t} p(s)\right|_{*, h}+\chi^{-1} \ell_{\Omega}\left\|\partial_{t t t} p(s)-I_{\mathcal{T}}^{k+1}\left(\partial_{t t t} p(s)\right)\right\|_{\frac{1}{\kappa} ; \Omega^{\Gamma}}\right) d s,
\end{aligned}
$$

and $|\cdot|_{*, h}$ is defined in (35). Moreover, we have

$$
\left\|\partial_{t} p_{\mathcal{T}}-\partial_{t} p\right\|_{C^{0}\left(0, t ; L^{2}\left(\frac{1}{\kappa} ; \Omega\right)\right)}+\left\|\boldsymbol{G}_{\mathcal{T}}\left(\hat{p}_{h}\right)-\nabla p\right\|_{C^{0}\left(0, t ; L^{2}\left(\frac{1}{\rho} ; \Omega\right)\right)} \lesssim h^{\nu} \Phi(t, p),
$$

with $\Phi(t, p):=\rho_{\mathrm{b}}^{-\frac{1}{2}}\left(|p|_{L^{\infty}\left(0, t ; H^{1+\nu}(\Omega)\right)}+\left|\partial_{t} p\right|_{L^{1}\left(0, t ; H^{1+\nu}(\Omega)\right)}\right)+\kappa_{\mathrm{b}}^{-\frac{1}{2}} \chi^{-1} \ell_{\Omega} h\left(\left|\partial_{t t} p\right|_{L^{\infty}\left(0, t ; H^{1+\nu}(\Omega)\right)}+\right.$ $\left.\left|\partial_{t t t} p\right|_{L^{1}\left(0, t ; H^{1+\nu}(\Omega)\right)}\right)$. 
Proof. Step 1: Error equation. We observe that for all $\hat{q}_{h} \in \hat{V}_{h 0}$ and all $t \in J$,

$$
\begin{aligned}
\left(\partial_{t t} e_{\mathcal{T}}(t), q_{\mathcal{T}}\right)_{\frac{1}{\kappa} ; \Omega}+b_{h}\left(\hat{e}_{h}(t), \hat{q}_{h}\right) & =\left(f(t), q_{\mathcal{T}}\right)_{\Omega}-\left(\partial_{t t} I_{\mathcal{T}}^{k+1}(p(t)), q_{\mathcal{T}}\right)_{\frac{1}{\kappa} ; \Omega}-b_{h}\left(I_{h}^{k}(p(t)), \hat{q}_{h}\right) \\
& =\left(\partial_{t t} p(t)-I_{\mathcal{T}}^{k+1}\left(\partial_{t t} p(t)\right), q_{\mathcal{T}}\right)_{\frac{1}{\kappa} ; \Omega^{\Gamma}}+\delta_{h}\left(p(t) ; \hat{q}_{h}\right)=: \theta_{h}\left(t ; \hat{q}_{h}\right),
\end{aligned}
$$

where we used that $\left(\partial_{t t} p(t)-I_{\mathcal{T}}^{k+1}\left(\partial_{t t} p(t)\right), q_{\mathcal{T}}\right)_{\frac{1}{\kappa} ; \Omega \backslash \Omega^{\Gamma}}=0$ and where the linear form $\delta_{h}(p(t) ; \cdot)$ is defined in (33) and bounded in (34). Invoking the Cauchy-Schwarz inequality and Lemma 6.2 (see below) gives

$$
\left\|\theta_{h}(t ; \cdot)\right\|_{\left(\hat{V}_{h 0}\right)^{\prime}} \lesssim|p(t)|_{*, h}+\chi^{-1} \ell_{\Omega}\left\|\partial_{t t} p(t)-I_{\mathcal{T}}^{k+1}\left(\partial_{t t} p(t)\right)\right\|_{\frac{1}{\kappa} ; \Omega^{\Gamma}} .
$$

Step 2: Stability argument. We test the error equation with $\hat{q}_{h}=\partial_{t} \hat{e}_{h}(t)$ for all $t \in J$. Owing to the symmetry of $b_{h}$ and integrating by parts in time on the right-hand side, we infer that

$$
\frac{d}{d t}\left\{\frac{1}{2}\left\|\partial_{t} e_{\mathcal{T}}(t)\right\|_{\frac{1}{\kappa} ; \Omega}^{2}+\frac{1}{2} b_{h}\left(\hat{e}_{h}(t), \hat{e}_{h}(t)\right)\right\}=\frac{d}{d t} \theta_{h}\left(t ; \hat{e}_{h}(t)\right)-\dot{\theta}\left(t ; \hat{e}_{h}(t)\right),
$$

where we have set $\dot{\theta}(t ; \cdot):=\delta_{h}\left(\partial_{t} p(t) ; \cdot\right)+\left(\partial_{t t t} p(t)-I_{\mathcal{T}}^{k+1}\left(\partial_{t t t} p(t)\right), \cdot\right)_{\frac{1}{\kappa} ; \Omega^{\Gamma}}$, so that

$$
\left\|\dot{\theta}_{h}(t ; \cdot)\right\|_{\left(\hat{V}_{h 0}\right)^{\prime}} \lesssim\left|\partial_{t} p(t)\right|_{*, h}+\chi^{-1} \ell_{\Omega}\left\|\partial_{t t t} p(t)-I_{\mathcal{T}}^{k+1}\left(\partial_{t t t} p(t)\right)\right\|_{\frac{1}{\kappa} ; \Omega^{\Gamma}} .
$$

Integrating in time from 0 to $t$, observing that $\partial_{t} e_{\mathcal{T}}(0)=0$ owing to the initial conditions satisfied by $\hat{p}_{h}$, and using the coercivity property (28) gives

$$
\frac{1}{2}\left\|\partial_{t} e_{\mathcal{T}}(t)\right\|_{\frac{1}{\kappa} ; \Omega}^{2}+\frac{1}{2} \alpha\left\|\hat{e}_{h}(t)\right\|_{\hat{V}_{h 0}}^{2} \leq \theta_{h}\left(t ; \hat{e}_{h}(t)\right)-\theta_{h}\left(0 ; \hat{e}_{h}(0)\right)-\int_{0}^{t} \dot{\theta}_{h}\left(s ; \hat{e}_{h}(s)\right) d s+C\left\|\hat{e}_{h}(0)\right\|_{\hat{V}_{h 0}}^{2},
$$

where the last term results from the boundedness of the discrete bilinear form $b_{h}$ on $\hat{V}_{h 0} \times \hat{V}_{h 0}$. Reasoning as in the proof of [9, Thm. 3.1] leads to

$$
\begin{aligned}
& \frac{1}{2}\left\|\partial_{t} e_{\mathcal{T}}\right\|_{C^{0}\left(0, t ; L^{2}\left(\frac{1}{\kappa} ; \Omega\right)\right)}^{2}+\frac{1}{8} \alpha\left\|\hat{e}_{h}\right\|_{C^{0}\left(0, t ; \hat{V}_{h 0}\right)}^{2} \\
& \quad \leq C\left(\left|\theta_{h}\right|_{C^{0}\left(0, t ;\left(\hat{V}_{h 0}\right)^{\prime}\right)}^{2}+\left|\dot{\theta}_{h}\right|_{L^{1}\left(0, t ;\left(\hat{V}_{h 0}\right)^{\prime}\right)}^{2}+\left\|\hat{e}_{h}(0)\right\|_{\hat{V}_{h 0}}^{2}\right),
\end{aligned}
$$

with $\left|\theta_{h}\right|_{C^{0}\left(0, t ;\left(\hat{V}_{h 0}\right)^{\prime}\right)}:=\sup _{s \in[0, t]}\left\|\theta_{h}(s ; \cdot)\right\|_{\left(\hat{V}_{h 0}\right)^{\prime}}$ and $\left|\dot{\theta}_{h}\right|_{L^{1}\left(0, t ;\left(\hat{V}_{h 0}\right)^{\prime}\right)}:=\int_{0}^{t}\left\|\dot{\theta}_{h}(s ; \cdot)\right\|_{\left(\hat{V}_{h 0}\right)^{\prime}} d s$. Still reasoning as in this proof (where one uses $e_{\mathcal{T}}(0)=0$ ) shows that $\left\|\hat{e}_{h}(0)\right\|_{\hat{V}_{h 0}} \lesssim\left|p_{0}\right|_{*, h}$. Combining these bounds with the estimates (41)-(42) proves (37).

Step 3: Convergence rates. The estimate (40) follows from (37) and the triangle inequality, after invoking the approximation properties (31)-(32) and those of the gradient reconstruction from [6, Lem. 3.8]. Notice that the decay rates are optimal if $\nu=k+1$.

Lemma 6.2 (Poincaré inequality). We have $\left\|q_{\mathcal{T}}\right\|_{\Omega} \lesssim \rho_{\sharp}^{\frac{1}{2}} \ell_{\Omega}\left\|\hat{q}_{h}\right\|_{\hat{V}_{h 0}}$ for all $\hat{q}_{h} \in \hat{V}_{h 0}$.

Proof. Let $\hat{q}_{h} \in \hat{V}_{h 0}$. There is $\boldsymbol{v} \in \boldsymbol{H}^{1}(\Omega)$ such that $\nabla \cdot \boldsymbol{v}=q_{\mathcal{T}}$ and $\|\boldsymbol{v}\|_{\boldsymbol{H}^{1}(\Omega)}:=\left(\|\boldsymbol{v}\|_{\Omega}^{2}+\right.$ $\left.\ell_{\Omega}^{2}\|\nabla v\|_{\Omega}^{2}\right)^{\frac{1}{2}} \lesssim \ell_{\Omega}\left\|q_{\mathcal{T}}\right\|_{\Omega}$. Integrating by parts cellwise and re-arranging the terms shows that

$$
\begin{aligned}
\left\|q_{\mathcal{T}}\right\|_{\Omega}^{2}=\left(q_{\mathcal{T}}, \nabla \cdot \boldsymbol{v}\right)_{\Omega}= & \sum_{T \in \mathcal{T}_{h}} \sum_{i \in\{1,2\}}\left\{-\left(\nabla q_{T^{i}}, \boldsymbol{v}\right)_{T^{i}}+\left(q_{T^{i}}-q_{(\partial T)^{i}}, \boldsymbol{v} \cdot \boldsymbol{n}_{T}\right)_{(\partial T)^{i}}\right\} \\
& +\sum_{T \in \mathcal{T}_{h}}\left(\llbracket q_{T} \rrbracket_{\Gamma}, \boldsymbol{v} \cdot \boldsymbol{n}_{\Gamma}\right)_{T^{\Gamma}},
\end{aligned}
$$


where we used that $\boldsymbol{v}$ is single-valued at all the mesh interfaces and that $q_{F}$ vanishes at all the mesh boundary faces. Recalling the definition (27) of the norm $\|\cdot\|_{\hat{V}_{h 0}}$ and invoking the Cauchy-Schwarz inequality gives

$$
\left\|q_{\mathcal{T}}\right\|_{\Omega}^{2} \lesssim \rho_{\sharp}^{\frac{1}{2}}\left\|\hat{q}_{h}\right\|_{\hat{V}_{h 0}}\left(\sum_{T \in \mathcal{T}_{h}} \sum_{i \in\{1,2\}}\|\boldsymbol{v}\|_{T^{i}}^{2}+h_{T}\|\boldsymbol{v}\|_{\partial\left(T^{i}\right)}^{2}\right)^{\frac{1}{2}} .
$$

To bound the last term on the right-hand side, we invoke the multiplicative trace inequality (6) (applied componentwise) followed by Young's inequality to infer that $h_{T}^{\frac{1}{2}}\|\boldsymbol{v}\|_{\partial\left(T^{i}\right)} \lesssim\|\boldsymbol{v}\|_{T^{\dagger}}+$ $h_{T}\|\nabla \boldsymbol{v}\|_{T^{\dagger}}$ for all $T \in \mathcal{T}_{h}$ and all $i \in\{1,2\}$. This yields

$$
\|q \mathcal{T}\|_{\Omega}^{2} \lesssim \rho_{\sharp}^{\frac{1}{2}}\left\|\hat{q}_{h}\right\|_{\hat{V}_{h 0}}\left(\sum_{T \in \mathcal{T}_{h}}\|\boldsymbol{v}\|_{T^{\dagger}}^{2}+h_{T}^{2}\|\nabla \boldsymbol{v}\|_{T^{\dagger}}^{2}\right)^{\frac{1}{2}} .
$$

Since $h_{T} \leq \ell_{\Omega}$ and owing to Assumption 3.3, we have

$$
\sum_{T \in \mathcal{T}_{h}}\|\boldsymbol{v}\|_{T^{\dagger}}^{2}+h_{T}^{2}\|\nabla \boldsymbol{v}\|_{T^{\dagger}}^{2} \lesssim\|\boldsymbol{v}\|_{\boldsymbol{H}^{1}(\Omega)}^{2},
$$

and we conclude by recalling that $\|\boldsymbol{v}\|_{\boldsymbol{H}^{1}(\Omega)} \lesssim \ell_{\Omega}\left\|q_{\mathcal{T}}\right\|_{\Omega}$.

Remark 6.3 (Theorem 6.1). The difference in the initial condition between (18) and Theorem 6.1 only concerns the cut cells $T \in \mathcal{T}_{h}^{\Gamma}$ (where the $L^{2}$-orthogonal projection uses $T$ and $T^{\dagger}$ respectively). This choice is a consequence of the use of the error decomposition (36) which implies that $e_{\mathcal{T}}(t)=p_{\mathcal{T}}(t)-I_{\mathcal{T}}^{k+1}(p(t))$ which possibly differs from $p_{\mathcal{T}}(t)-\Pi_{\mathcal{T}}^{k+1}(p(t))$ in the cut cells, and in turn, this error decomposition is motivated by the approximation properties (31)(32). Using $I_{\mathcal{T}}^{k+1}$ in lieu of $\Pi_{\mathcal{T}}^{k+1}$ is also at the origin of the need to invoke the Poincaré inequality from Lemma 6.2 (leading to the velocity factor $\chi$ ) and the assumption on the third-order time derivative of $p$ (instead of the second-order only). Notice though that the perturbation induced on the factor $\Phi(t, p)$ in (40) is of higher order. We believe that these are artifacts of the theoretical analysis, but leave further exploration of these aspects to future work.

\section{Numerical results}

In this section, we discuss our numerical results. For the second-order formulation in time (see Section 4), the time discretization is based on the implicit, second-order accurate Newmark scheme with parameters $(\beta, \gamma)=\left(\frac{1}{4}, \frac{1}{2}\right)$. For the first-order formulation in time (see Section 4 ), the time discretization is based on Runge-Kutta (RK) schemes up to fourth-order accuracy. We consider both singly-diagonally implicit schemes with $s$ stages and order $(s+1)$ with $s \in\{1,2,3\}$ (in short, $\operatorname{SDIRK}(s, s+1)$ ) and explicit schemes with $s$ stages and order $s$ with $s \in\{2,3,4\}$ (in short, $\operatorname{ERK}(s)$ ). The Butcher tableaux of the RK schemes and their algebraic realization are documented in [8]. Notice that the Newmark scheme is always applied to the second-order formulation in time of the wave equation, whereas the RK schemes are applied to the first-order formulation.

\subsection{Implementation}

The implementation of fitted HHO methods is discussed in [12]. Here, we focus on implementation aspects related to the unfitted setting. 

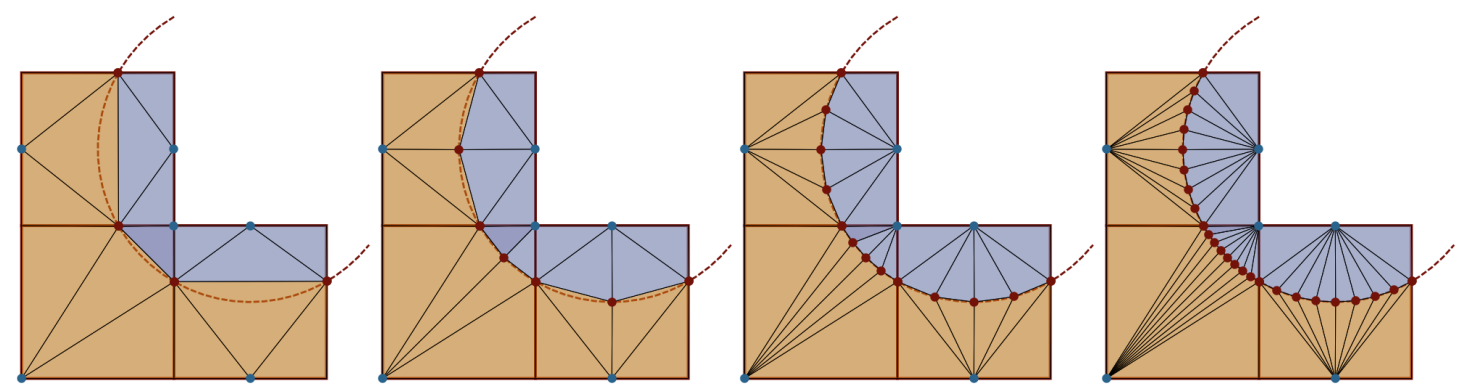

Figure 2: Geometric discretization of the interface and the sub-cells in cut cells. The number of points discretizing the interface is $2^{n_{\text {int }}}+1$ with $n_{\text {int }} \in\{0,1,2,3\}$ (left to right).

First, some preprocessing operations are performed on the given Cartesian mesh (background mesh): tagging each geometric entity with an identifier to state whether the object is in $\Omega_{1}$ or $\Omega_{2}$; searching for those cells and interfaces that are cut by the interface $\Gamma$; identifying the cells with a poor cut and performing a local cell-agglomeration procedure. The agglomeration algorithm is detailed in [6, Sec. 5.1] and uses a threshold parameter $\theta_{\text {agg. }}$. The cell is flagged as being poorly cut if the ratio between the area of each sub-cell and the area of the cell falls below the threshold. Unless stated otherwise, we set $\theta_{\text {agg }}:=0.3$ in our numerical experiments.

To perform numerical integration in the cut cells and along the interface, a set of points is first constructed to discretize the interface as a piecewise affine curve. This set of points is defined by means of a parameter $n_{\text {int }}$ so that there are $2^{n_{\text {int }}}+1$ points discretizing the interface in each cell. Using these points, a sub-triangulation is created in each sub-cell. Finally, the quadrature points and weights are obtained by gathering all the Dunavant [19] quadrature points and weights for all resulting sub-triangles. Figure 2 shows how the sub-triangulation is constructed when $n_{\text {int }} \in\{0,1,2,3\}$. Unless stated otherwise, we use $n_{\text {int }}:=8$ in our numerical experiments.

\subsection{Convergence tests for implicit schemes}

In this section, we verify the convergence rates delivered by the implicit schemes (Newmark and SDIRK) on smooth solutions in homogeneous media. We also assess the impact of geometry discretization errors. We consider the two-dimensional domain $\Omega:=(0,1)^{2}$, the final time $T_{\mathrm{f}}:=1$, and the material properties are $\rho:=\kappa:=1$. The material interface is defined by a circular level set function:

$$
\phi_{c}(x, y):=\left(x-x_{c}\right)^{2}+\left(y-y_{c}\right)^{2}-r^{2},
$$

with $r:=\frac{1}{3}$ and $x_{c}=y_{c}:=\frac{1}{2}$. Two smooth solutions are considered:

$$
\begin{aligned}
& p(t, x, y)=t^{2} \sin (\pi x) \sin (\pi y), \\
& p(t, x, y)=\sin (\sqrt{2} \pi t) x(1-x) y(1-y),
\end{aligned}
$$

where (44) is used to focus on the space discretization error and (45) on the time discretization error. In both cases, the data $f$ and the initial conditions $p_{0}$, and $v_{0}$ are computed accordingly. We use a sequence of uniformly refined quadrangular meshes of size $h:=0.1 \times 2^{-l}$ with $l \in$ $\{0,1,2,3,4\}$, and the time step size is set to $\Delta t:=0.1 \times 2^{-l}$ with $l \in\{0,1,2,3,4,5\}$. In the following convergence tests, we report the $H^{1}$ - and $L^{2}$-errors at the final time. Figure 3 (upper row) reports the convergence in space for the analytical solution (44) and the convergence in time for the analytical solution (45) using the Newmark scheme. The convergence orders in 

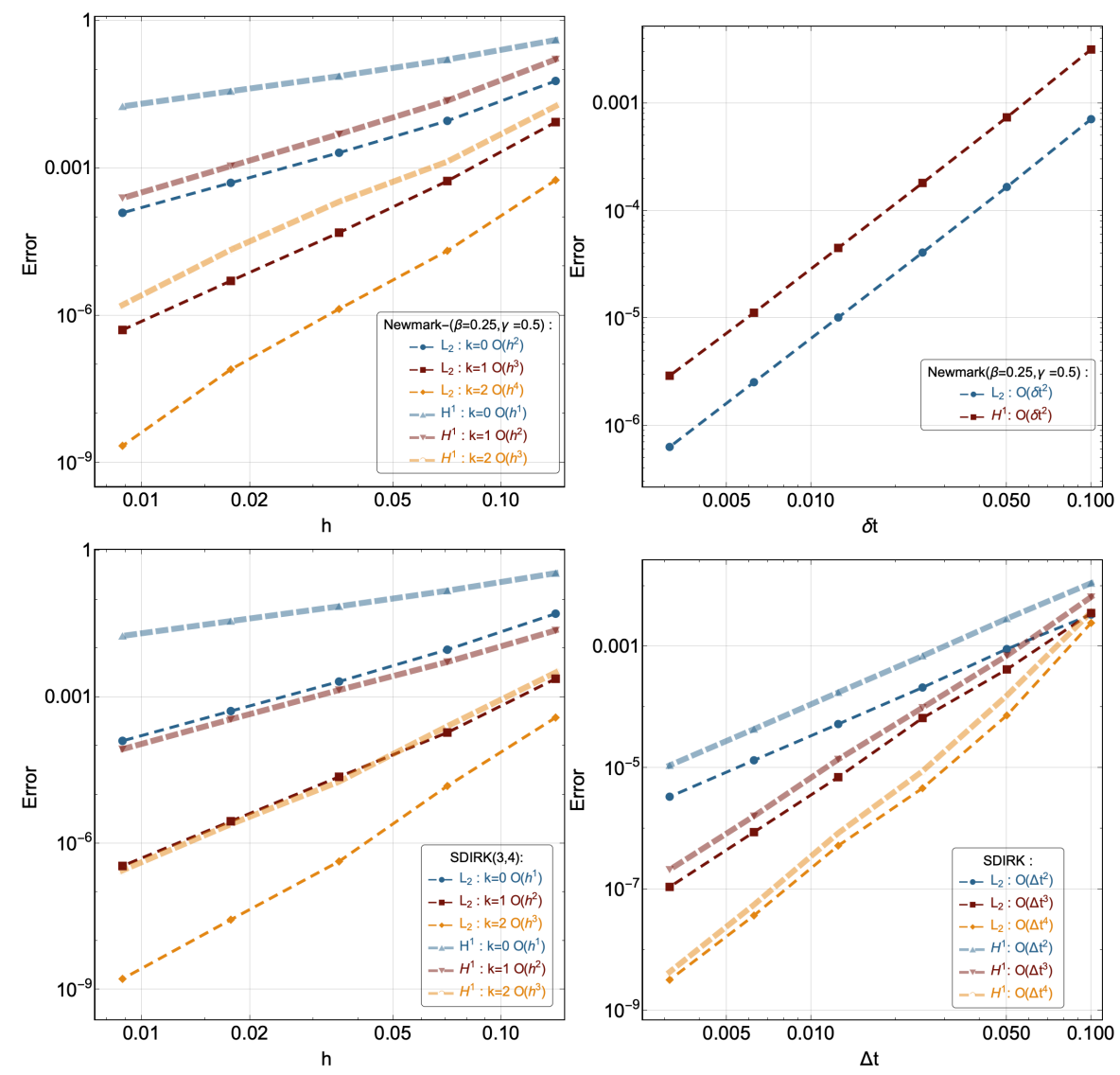

Figure 3: Convergence in space for the analytical solution (44) (left panels), and convergence in time for the analytical solution (45) (right panels). Top row: Newmark scheme; bottom row: $\operatorname{SDIRK}(s, s+1)$ scheme with $s=3$ in the bottom left panel to study the convergence in space and $s \in\{1,2,3\}$ in the bottom right panel to study the convergence in time $\left(n_{\text {int }}:=10\right)$.

space match the theoretical predictions for smooth solutions, while the convergence in time is of second order as expected. The same conclusions are reached when considering SDIRK schemes (Figure 3, bottom row). Next, we explore the effect of the geometry discretization parameter $n_{\text {int }}$ in Figure 4, both for the Newmark and SDIRK schemes. The results indicate that for the values $n_{\text {int }}=\{8,10\}$, the geometric error is negligible compared to the time discretization error for the current choices of the time step. Similar experiments (not reported herein for brevity, see, e.g., [6, Fig. 5.6] for a study on the elliptic interface problem) indicate that given a range for the mesh size, the parameter $n_{\text {int }}$ has to be taken large enough so that the geometric error does not dominate the space discretization error.

\subsection{Fitted-unfitted comparison: heterogeneous case with flat interface}

The second test case deals with the propagation of an acoustic wave in a two-dimensional heterogeneous domain with a flat interface, so as to allow for the comparison between the fitted and unfitted HHO methods. The domain is $\Omega:=\left(-\frac{3}{2}, \frac{3}{2}\right)^{2}$, the simulation time is $T_{\mathrm{f}}:=1$, the inter- 

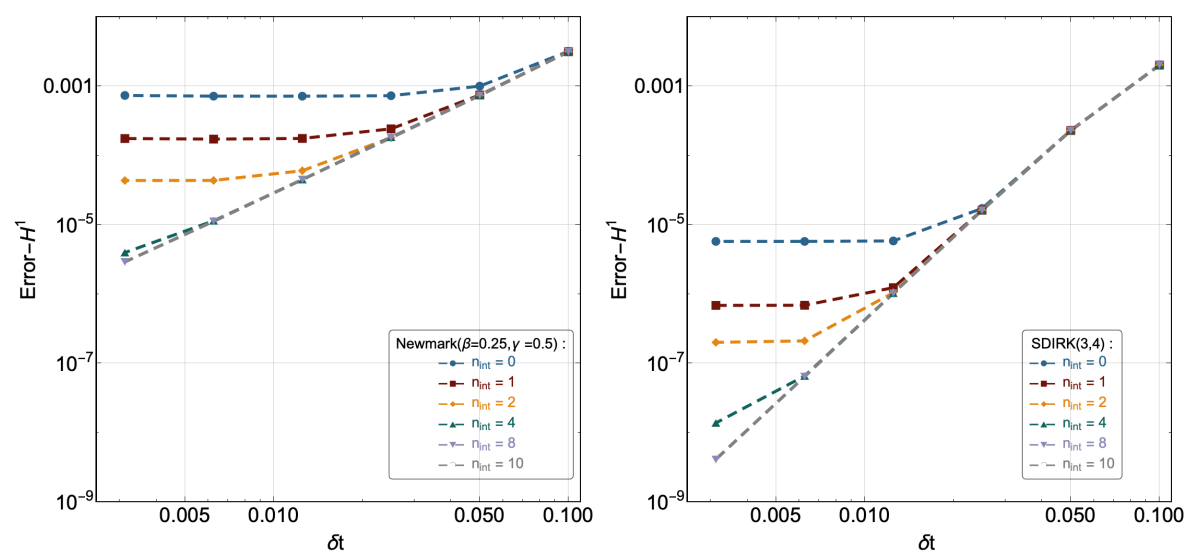

Figure 4: Impact of the choice of the geometry discretization parameter $n_{\text {int }}$ in the convergence in time for the analytical solution (45); Newmark scheme (left panel) and $\operatorname{SDIRK}(3,4)$ scheme (right panel).

face is a line located at $y=0, \Omega_{1}$ is located below the interface and $\Omega_{2}$ above. We consider two cases for the material properties: the low-contrast case where $\kappa_{1}:=3, \kappa_{2}:=9$ and $\rho_{1}=\rho_{2}=1$ (so that the velocity ratio is $c_{2} / c_{1}=\sqrt{3}$ ), and the high-contrast case where $\kappa_{1}:=3, \kappa_{2}:=192$ and $\rho_{1}=\rho_{2}=1$ (so that the velocity ratio is $c_{2} / c_{1}=8$ ). The source term is $f:=0$, and the initial conditions are $v_{0}:=0$ and

$$
p_{0}(x, y):=\theta \exp \left(-\pi^{2} \frac{r^{2}}{\lambda^{2}}\right)
$$

with $\lambda:=\frac{c_{2}}{\alpha}, \alpha:=10$ for the low-contrast case and $\alpha:=20$ for the high-contrast case, $\theta:=\alpha \frac{\lambda^{2}}{2 \pi^{2}}$, $r^{2}:=\left(x-x_{c}\right)^{2}+\left(y-y_{c}\right)^{2}, x_{c}:=0$, and $y_{c}:=\frac{2}{3}$. The initial condition corresponds to a Ricker wave centered at the point $\left(x_{c}, y_{c}\right) \in \Omega_{2}$. The wave first propagates in $\Omega_{2}$, then is partially transmitted to $\Omega_{1}$ and later it is also reflected at the boundary of $\Omega$. A semi-analytical solution is available until the wave is reflected at the boundary of $\Omega$, and can be calculated using the gar6more2d software. ${ }^{1}$ The semi-analytical solution is based on a reformulation of the problem with zero initial conditions and a Dirac source term with a time delay of 0.15 (this value is tuned to match the choice of the parameter $\theta$ above).

In our comparisons between the numerical predictions using HHO and the semi-analytical solution, we track the two Cartesian components of $\sigma=\frac{1}{\rho} \nabla p$ at two sensors, one located at the point $S_{1}:=\left(\frac{3}{4},-\frac{1}{3}\right)$ in $\Omega_{1}$ and one located at the point $S_{2}:=\left(\frac{3}{4}, \frac{1}{3}\right)$ in $\Omega_{2}$. The comparison with the semi-analytical solution remains valid until the waves reflected at $\partial \Omega$ reach one of the sensors (this happens at about $t_{1}^{*} \approx 0.8$ for $S_{1}$ and $t_{2}^{*} \approx 0.6$ for $S_{2}$ in the low-contrast case and at about $t_{1}^{*} \approx 0.3$ for $S_{1}$ and $t_{2}^{*} \approx 0.1$ for $S_{2}$ in the high-contrast case). For the numerical predictions, we compare the fitted HHO method from [8] (termed Fit-HHO in the plots) and the unfitted HHO method developed herein (termed Cut-HHO in the plots). The size of the fitted mesh is $h=\frac{1}{32}$ or $h=\frac{1}{64}$, and we consider the polynomial degrees $k \in\{1,2,3\}$. In the low-contrast case, we always use $h=\frac{1}{32}$, whereas in the high-contrast case, we always use $h=\frac{1}{32}$ for the Newmark scheme and we compare both mesh sizes for $\operatorname{SDIRK}(3,4)$. The unfitted mesh is created by agglomerating the 32 or 64 cells located just above and just below the interface. Since the interface is flat,

\footnotetext{
${ }^{1}$ see https://gforge.inria.fr/projects/gar6more2d/
} 

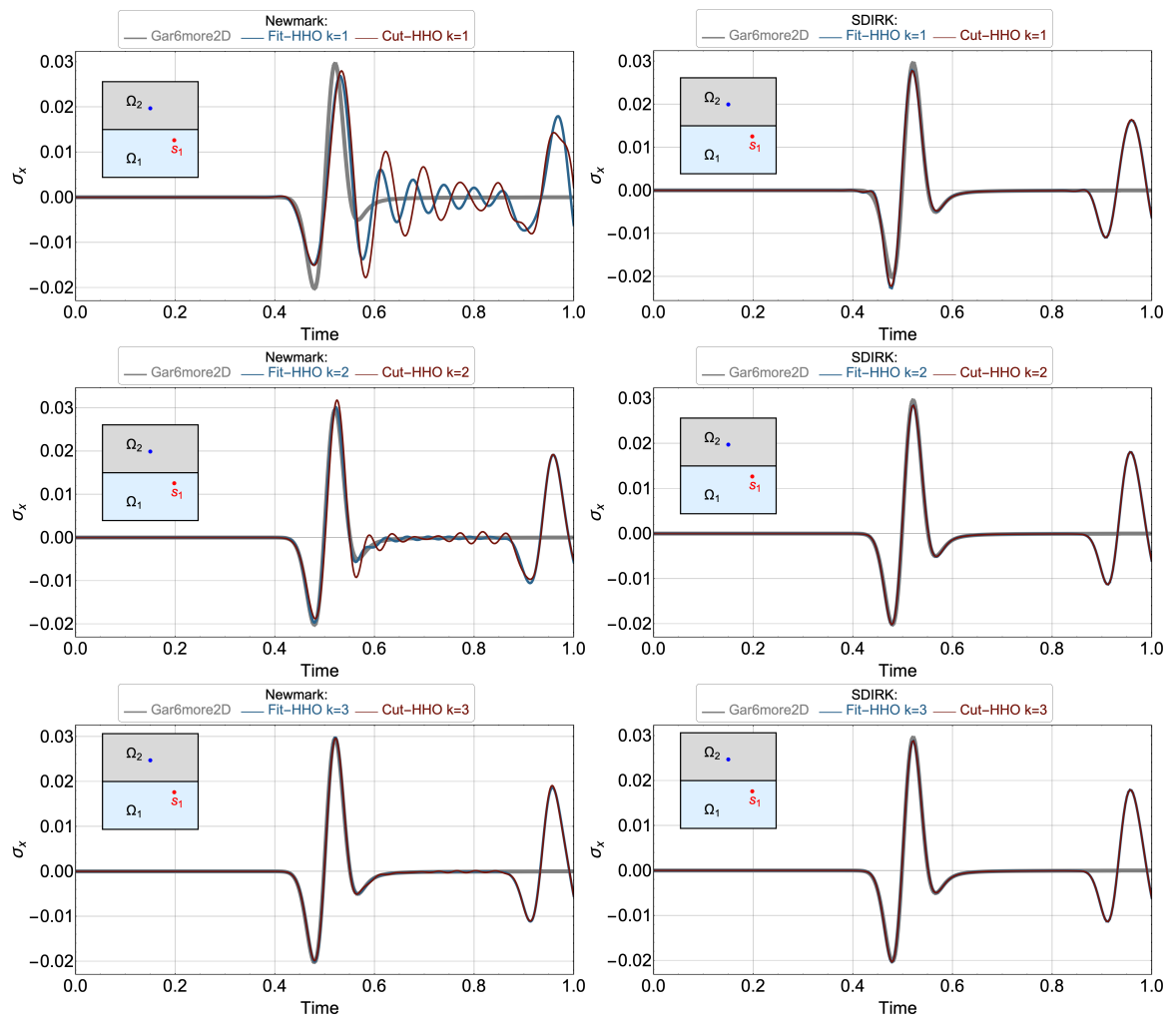

Figure 5: Low-contrast test case. Comparison of the signal $\sigma_{x}$ at the sensor $S_{1}$ obtained using the semi-analytical solution, the fitted HHO method (Fit-HHO), and the unfitted HHO method (Cut-HHO). Left column: Newmark time-stepping; right column: $\operatorname{SDIRK}(3,4)$ time-stepping. Top row: $k=1$; central row: $k=2$; bottom row: $k=3$. $h=\frac{1}{32}$ for both schemes.

we can take $n_{\text {int }}=0$. For the signal at the sensors, we use the reconstructed gradient in the second-order formulation, and the cell component of $\boldsymbol{\sigma}_{\mathcal{T}}$ in the first-order formulation. The time discretization employs either the Newmark scheme (time steps $\Delta t=0.1 \times 2^{-7}$ for low contrast and $\Delta t=0.1 \times 2^{-9}$ for high contrast) or the $\operatorname{SDIRK}(3,4)$ scheme (time step $\Delta t=0.1 \times 2^{-5}$ for low contrast and $\Delta t=0.1 \times 2^{-6}$ for high contrast).

The results are reported in Figure 5 (low contrast) and in Figure 6 (high contrast) for the $x$-component at the sensor $S_{1}$ (the conclusions are similar for the $y$-component and for the signals recorded at $S_{2}$ ). We observe in both cases (low and high contrast) that for the Newmark scheme, the choice $k=1$ for the polynomial order leads to fairly large errors. The situation is improved for $k=2$, although some oscillations remain, whereas the agreement with the semi-analytical solution is excellent for $k=3$. We also notice that the results obtained with the fitted HHO method present somewhat less pronounced oscillations than with the unfitted HHO method, although the differences are not very significant and disappear by increasing the polynomial degree. Concerning $\operatorname{SDIRK}(3,4)$, the accuracy is always very good in the low-contrast case. In the high-contrast case, when using the coarser mesh with $h=\frac{1}{32}$, the signals for $k \in\{1,2\}$ feature some spurious oscillations (both for the fitted and unfitted approaches), but these oscillations disappear for $k=3$. Additional numerical experiments (not shown here for brevity, see Table 2) indicate that the results are already very accurate for $k=1$ when using the finer mesh with 

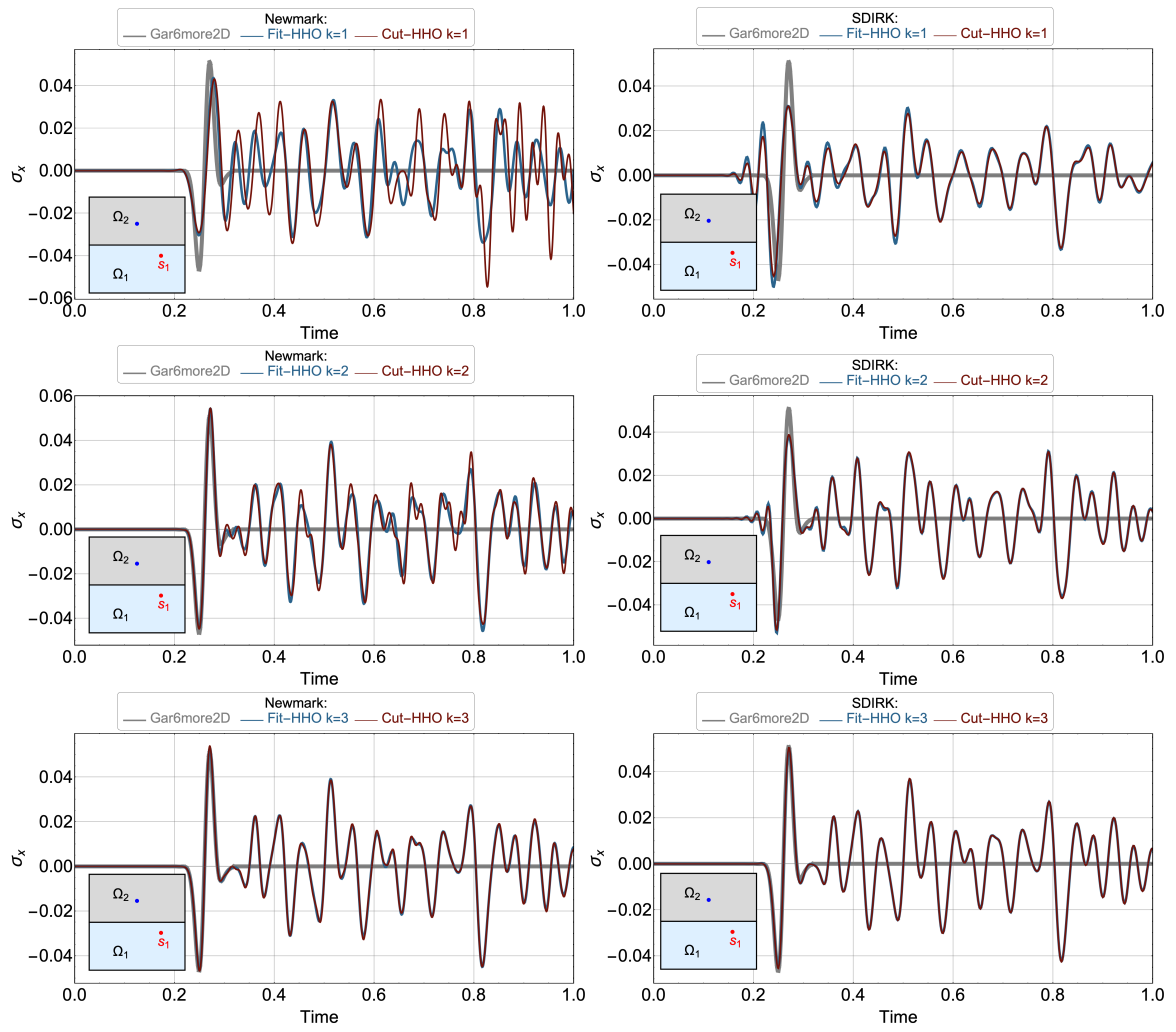

Figure 6: High-contrast test case. Comparison of the signal $\sigma_{x}$ at the sensor $S_{1}$ obtained using the semi-analytical solution, the fitted HHO method (Fit-HHO), and the unfitted HHO method (Cut-HHO). Left column: Newmark time-stepping; right column: SDIRK $(3,4)$ time-stepping. Top row: $k=1$; central row: $k=2$; bottom row: $k=3 . h=\frac{1}{64}$ for Newmark and $h=\frac{1}{32}$ for $\operatorname{SDIRK}(3,4)$.

$h=\frac{1}{64}$.

A more quantitative comparison is provided in Table 1 (low contrast) and in Table 2 (high contrast), where we report the maximum relative error (in \%) for both components of $\boldsymbol{\sigma}$ over all the discrete time nodes in the time interval $\left[0, t_{i}^{*}\right]$ for the sensor $S_{i}, i \in\{1,2\}$. The normalization is computed by using the maximum values in time (in absolute value) obtained for the semianalytical solution: the resulting values are $2.95 \times 10^{-2}$ for $\sigma_{x}$ at $S_{1}, 7.05 \times 10^{-2}$ for $\sigma_{y}$ at $S_{1}$, $7.13 \times 10^{-2}$ for $\sigma_{x}$ at $S_{2}$, and $3.17 \times 10^{-2}$ for $\sigma_{y}$ at $S_{2}$ in the low-contrast case, and $5.11 \times 10^{-2}$ for $\sigma_{x}$ at $S_{1}, 5.98 \times 10^{-1}$ for $\sigma_{y}$ at $S_{1}, 5.65 \times 10^{-1}$ for $\sigma_{x}$ at $S_{2}$, and $2.26 \times 10^{-1}$ for $\sigma_{y}$ at $S_{2}$ in the high-contrast case. We observe again the significant improvement gained by increasing the polynomial degree. Moreover, we see that in all cases, the errors incurred with the fitted and unfitted HHO method are comparable, with slightly better results for the fitted version. Observe though that the unfitted mesh is produced by agglomerating two adjacent cells, so that the cells used in the unfitted HHO method are actually two times larger around the interface. 


\begin{tabular}{|c|c|c|c|c|c|c|}
\hline Newmark & \multicolumn{3}{|c|}{ Fit-HHO } & \multicolumn{3}{c|}{ Cut-HHO } \\
\hline Variable and location & $k=1$ & $k=2$ & $k=3$ & $k=1$ & $k=2$ & $k=3$ \\
\hline \hline$\sigma_{x}$ at $S_{1}$ & 41.43 & 6.81 & 3.90 & 57.07 & 20.18 & 4.76 \\
\hline$\sigma_{y}$ at $S_{1}$ & 42.85 & 7.28 & 4.08 & 65.02 & 23.77 & 5.70 \\
\hline$\sigma_{x}$ at $S_{2}$ & 12.14 & 3.52 & 3.34 & 23.77 & 6.38 & 3.43 \\
\hline$\sigma_{y}$ at $S_{2}$ & 10.76 & 3.34 & 3.33 & 33.18 & 6.36 & 3.32 \\
\hline
\end{tabular}

\begin{tabular}{|c|c|c|c|c|c|c|}
\hline SDIRK $(3,4)$ & \multicolumn{3}{|c|}{ Fit-HHO } & \multicolumn{3}{c|}{ Cut-HHO } \\
\hline Variable and location & $k=1$ & $k=2$ & $k=3$ & $k=1$ & $k=2$ & $k=3$ \\
\hline \hline$\sigma_{x}$ at $S_{1}$ & 12.91 & 3.67 & 2.70 & 12.32 & 3.60 & 2.67 \\
\hline$\sigma_{y}$ at $S_{1}$ & 11.89 & 3.54 & 2.54 & 10.09 & 3.73 & 2.52 \\
\hline$\sigma_{x}$ at $S_{2}$ & 3.12 & 1.48 & 1.37 & 3.14 & 1.45 & 1.38 \\
\hline$\sigma_{y}$ at $S_{2}$ & 2.29 & 1.61 & 1.41 & 2.42 & 1.57 & 1.41 \\
\hline
\end{tabular}

Table 1: Low-contrast case. Maximum relative errors (in \%) for the components $\sigma_{x}$ and $\sigma_{y}$ at the two sensors $S_{1}$ and $S_{2}$; upper table: Newmark time-stepping; lower table: $\operatorname{SDIRK}(3,4)$ time-stepping. $h=\frac{1}{32}$ for both schemes.

\subsection{CFL condition for explicit time-stepping}

The use of an $\operatorname{ERK}(s)$ scheme for time-stepping is subject to a CFL stability condition. The goal of this section is to explore this condition. First, we observe that the condition takes the general form

$$
c_{\sharp} \frac{\Delta t}{h^{\gamma}} \leq \beta(s) \mu(k),
$$

where $c_{\sharp}$ is the largest velocity in the medium, $\gamma \in\{1,2\}, \beta(s) \approx \mathcal{O}(1)$ is a coefficient that depends on the number of stages, and $\mu(k)$ is a coefficient that depends on the mesh geometry and the polynomial degree $k$ (and is expected to scale as $(k+1)^{-1}$ ). To compute the coefficient $\mu(k)$, we compute numerically on various meshes the largest eigenvalue, say $\lambda_{\max }(h, k)$, of the generalized spectral problem $A X=\lambda M X$, where $A$ and $M$ are the stiffness and mass matrices appearing in the algebraic realization (25) of the space semi-discrete HHO formulation with polynomial degree $k \geq 0$. The exponent $\gamma$ and the coefficient $\mu(k)$ are then determined so that $\mu(k) \approx h^{-\gamma} \lambda_{\max }(h, k)^{-1}$ is $h$-independent.

In this section, we take $\Omega:=(0,1)^{2}$ and uniform material properties. We consider uniform Cartesian meshes of size $h=0.1 \times 2^{-l}$ with $l \in\{0,1,2,3,4\}$. First, we consider a flat interface as in the previous section and compare the values obtained for the fitted and unfitted HHO methods. In both cases, our results show (somewhat expectedly) that $\gamma=1$ if the stabilization weight is $\tilde{\tau}_{\partial T}=\mathcal{O}(1)$ and $\gamma=2$ if the stabilization weight is $\tilde{\tau}_{\partial T}=\mathcal{O}\left(h^{-1}\right)$ (see Section 5 for the definition of the weights). This result corroborates that using $\tilde{\tau}_{\partial T}=\mathcal{O}(1)$ is much more effective in the context of $\operatorname{ERK}(s)$ schemes than using $\tilde{\tau}_{\partial T}=\mathcal{O}\left(h^{-1}\right)$, and only the former value is used in the rest of this work. Table 3 reports the value of $\mu(k)$ obtained for the polynomial orders $k \in\{0,1,2,3\}$ for the fitted and unfitted HHO methods. The dependence on the polynomial order is close to $(k+1)^{-1}$ as expected. Moreover, the ratio of the values of $\mu(k)$ for fitted and unfitted HHO is moderate, confirming that the use of the unfitted approach does not really degrade the CFL condition on the time step. Next, we consider a circular interface. The first five panels in Figure 7 (from left to right, top to bottom) highlight the agglomerated cells on the five meshes considered (the agglomeration parameter is set to $\theta_{\text {agg }}=0.3$ ). The sixth panel (bottom right) reports the reciprocal of the maximum eigenvalue, $\lambda_{\max }(h, k)^{-1}$, on all 


\begin{tabular}{|c|c|c|c|c|c|c|}
\hline Newmark & \multicolumn{3}{|c|}{ Fit-HHO } & \multicolumn{3}{c|}{ Cut-HHO } \\
\hline Variable and location & $k=1$ & $k=2$ & $k=3$ & $k=1$ & $k=2$ & $k=3$ \\
\hline \hline$\sigma_{x}$ at $S_{1}$ & 2.64 & 0.65 & 0.35 & 3.10 & 0.67 & 0.41 \\
\hline$\sigma_{y}$ at $S_{1}$ & 28.01 & 6.31 & 3.76 & 30.55 & 5.64 & 4.25 \\
\hline$\sigma_{x}$ at $S_{2}$ & 2.61 & 0.87 & 0.43 & 1.62 & 0.74 & 0.38 \\
\hline$\sigma_{y}$ at $S_{2}$ & 9.63 & 4.58 & 1.35 & 10.87 & 4.71 & 1.23 \\
\hline
\end{tabular}

\begin{tabular}{|c|c|c|c|c|c|c|}
\hline SDIRK $(3,4)$ & \multicolumn{3}{|c|}{ Fit-HHO } & \multicolumn{3}{c|}{ Cut-HHO } \\
\hline Variable and location & $k=1$ & $k=2$ & $k=3$ & $k=1$ & $k=2$ & $k=3$ \\
\hline \hline$\sigma_{x}$ at $S_{1}$ & 0.4363 & 0.2423 & 0.0199 & 0.4179 & 0.1834 & 0.0192 \\
\hline$\sigma_{y}$ at $S_{1}$ & 6.1923 & 0.2929 & 0.1325 & 6.0324 & 0.2785 & 0.1281 \\
\hline$\sigma_{x}$ at $S_{2}$ & 0.1064 & 0.0034 & 0.0026 & 0.1075 & 0.0024 & 0.0016 \\
\hline$\sigma_{y}$ at $S_{2}$ & 0.0485 & 0.0135 & 0.0125 & 0.0460 & 0.0143 & 0.0125 \\
\hline
\end{tabular}

Table 2: High-contrast case. Maximum relative errors (in \%) for the components $\sigma_{x}$ and $\sigma_{y}$ at the two sensors $S_{1}$ and $S_{2}$; upper table: Newmark time-stepping; lower table: $\operatorname{SDIRK}(3,4)$ time-stepping. $h=\frac{1}{64}$ for both schemes.

\begin{tabular}{|c|cccc|}
\hline$k$ & 0 & 1 & 2 & 3 \\
\hline Fit-HHO & 0.118 & 0.0522 & 0.0338 & 0.0229 \\
\hline Cut-HHO & 0.0765 & 0.0373 & 0.0232 & 0.0159 \\
Ratio & 1.5 & 1.4 & 1.5 & 1.4 \\
\hline
\end{tabular}

Table 3: Coefficient $\mu(k)$ for polynomial orders $k \in\{0,1,2,3\}$ and the fitted HHO method (Fit-HHO), the unfitted HHO method (Cut-HHO) together with the ratio of the two values.

the meshes and for the polynomial orders $k \in\{0,1,2,3\}$. We observe that despite the slight irregularity of the agglomeration process as the meshes are refined, the reciprocal of the maximum eigenvalue essentially behaves as $h^{-1}$. The resulting value of $\mu(k)$ is reported in Table 4 for the agglomeration parameters $\theta_{\text {agg }} \in\{0.1,0.3,0.5\}$. As expected, there is a slight increase in $\mu(k)$ as the agglomeration parameter $\theta_{\text {agg }}$ is decreased since lower values of $\theta_{\text {agg }}$ favor the presence of cells with worse cuts. However, we see that the impact is moderate since, for instance, the value of $\mu(k)$ for $\theta_{\text {agg }}=0.1$ is only about 2.5 times worse than the value for $\theta_{\text {agg }}=0.5$. Interestingly, the value of the ratio is fairly independent of the polynomial degree. Another interesting result is reported in [35] for the elastic wave equation discretized in space using continuous cut finite elements with a Nitsche-based coupling; therein, stabilization based on least-squares penalty on the jumps of normal derivatives is introduced to temper bad cuts, and a cut-independent CFL condition is reported.

\subsection{Comparison of implicit and explicit schemes}

The goal of this test case is to compare the computational efficiency of the three time-stepping schemes (Newmark, $\operatorname{SDIRK}(3,4)$, and $\operatorname{ERK}(4)$ ) on a test case where the interface has a more complex shape than above. Specifically, we employ the following flower-like level set function:

$$
\phi_{f}(x, y):=(x-a)^{2}+(y-b)^{2}-r^{2}-c \cos \left(n_{c} \theta\right),
$$

with $a=b:=0, r:=1, c:=\frac{1}{5}$ and $n_{c}:=8$. For this test case, we consider the same physical settings as in Section 7.3 in the case of low contrast, except for the location of the initial pulse 

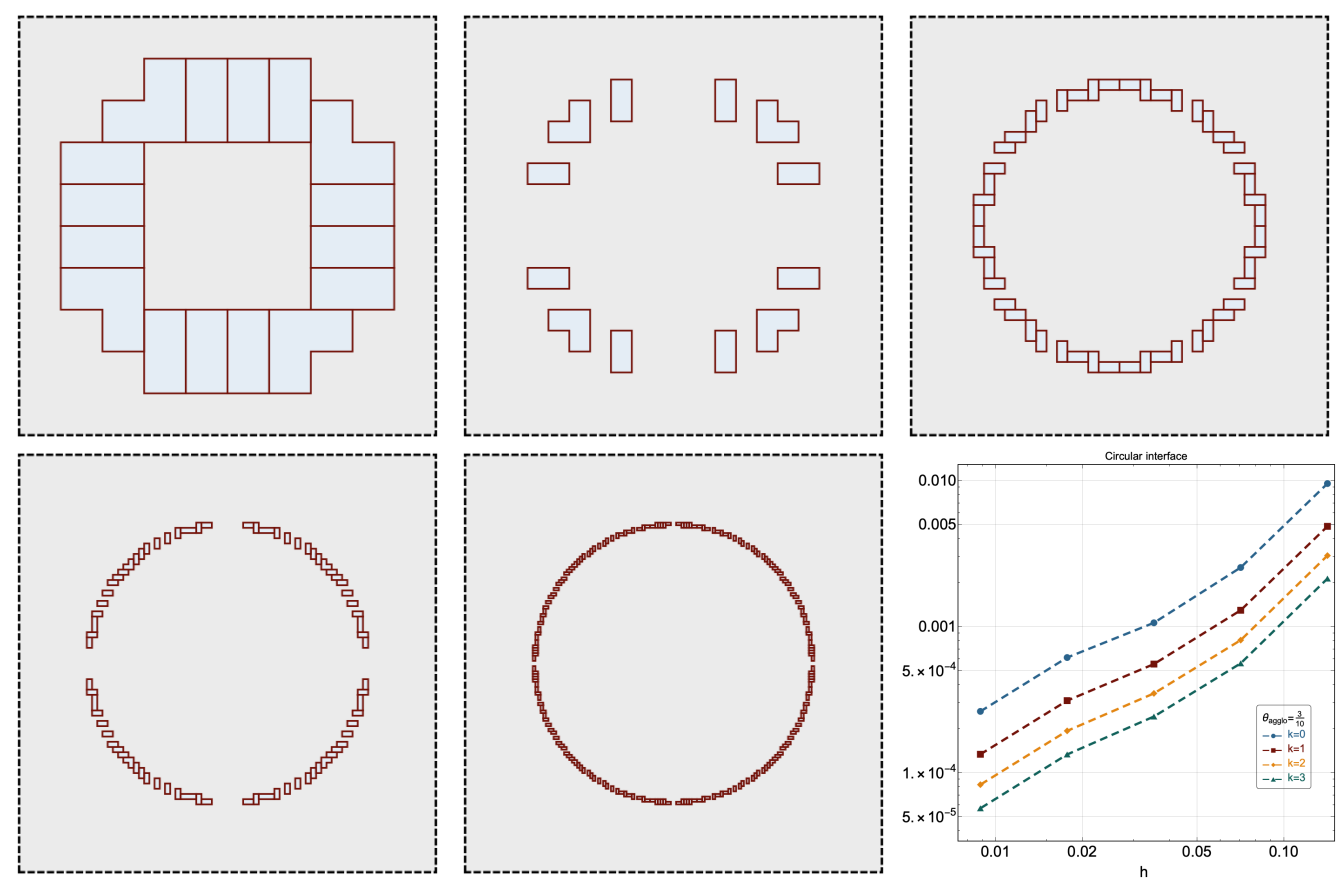

Figure 7: Agglomerated cells for the circular interface on a sequence of successively refined Cartesian meshes of size $h=0.1 \times 2^{-l}$ with $l \in\{0,1,2,3,4\}$ (from left to right, top to bottom). Bottom right panel: reciprocal of the maximum eigenvalue, $\lambda_{\max }(h, k)^{-1}$, on all the meshes and for the polynomial orders $k \in\{0,1,2,3\}$.

which is now $x_{c}=y_{c}:=0$. The sub-domain $\Omega_{2}$ is the one located inside the flower-like interface, whereas the sub-domain $\Omega_{1}$ is located outside. Since a semi-analytical solution is no longer available, we compute a reference solution by using $\operatorname{SDIRK}(3,4)$ time-stepping on a Cartesian mesh of size $h:=2^{-6}$, a time step $\Delta t:=0.1 \times 2^{-8}$, and polynomial order $k:=3$. Figure 8 shows the agglomerated cells on the meshes used in the numerical tests (left panel, $h:=2^{-5}$ ) and to compute the reference solution (right panel, $h:=2^{-6}$ ). Figure 9 illustrates the propagation of the wave across the flower-like interface and the reflections at the boundary. The selected time values are $t \in\{0.25,0.5,1.0\}$.

In order to compare the time-stepping schemes, we set the order of the HHO space semidiscretization to $k:=3$ and we select the value of the time-step for each implicit scheme in such a way that the accuracy of the signal corresponding to $\sigma_{x}$ and $\sigma_{y}$ at two sensors is of comparable accuracy. One sensor is located at the point $S_{1}:=(1.2,1)$ in $\Omega_{1}$ and the other one is located at the point $S_{2}:=\left(\frac{1}{3}, \frac{2}{3}\right)$ in $\Omega_{2}$. To refer to the level of time refinement, we write $\Delta t_{l}:=0.1 \times 2^{-l}$ with $l \in\{4, \ldots, 9\}$ and report the integer $l$. The mesh-size is $h:=2^{-5}$ for RK schemes and $h:=2^{-6}$ for the Newmark scheme; indeed, the space discretization errors tend to be a bit larger for the Newmark scheme since it handles second-order differential operators in space. Figure 10 reports the signal corresponding to $\sigma_{x}$ at the two sensors (similar results are obtained for $\sigma_{y}$ ) for the Newmark and the $\operatorname{SDIRK}(3,4)$ schemes with polynomial orders $k \in\{1,2,3\}$. These results corroborate the benefits of using a high polynomial degree (here, $k=3$ ). A more detailed overview of the errors for both signals at both sensors can be found in Table 5 which displays the maximum relative errors (in \%) over the time window $t \in[0,1]$ for the components $\sigma_{x}$ and 


\begin{tabular}{|c|cccc|}
\hline$k$ & 0 & 1 & 2 & 3 \\
\hline \hline$\theta_{\text {agg }}=0.5$ & 0.042 & 0.022 & 0.014 & 0.0099 \\
\hline$\theta_{\text {agg }}=0.3$ & 0.030 & 0.015 & 0.0094 & 0.0065 \\
Ratio & 1.4 & 1.5 & 1.5 & 1.5 \\
\hline$\theta_{\text {agg }}=0.1$ & 0.017 & 0.0087 & 0.0055 & 0.0039 \\
Ratio & 2.5 & 2.6 & 2.6 & 2.5 \\
\hline
\end{tabular}

Table 4: Coefficient $\mu(k)$ for polynomial orders $k \in\{0,1,2,3\}$ and the unfitted HHO method (Cut-HHO) for a circular interface and the agglomeration parameters $\theta_{\text {agg }} \in\{0.1,0.3,0.5\}$. The ratios are with respect to the value of $\mu(k)$ obtained with $\theta_{\text {agg }}=0.5$.
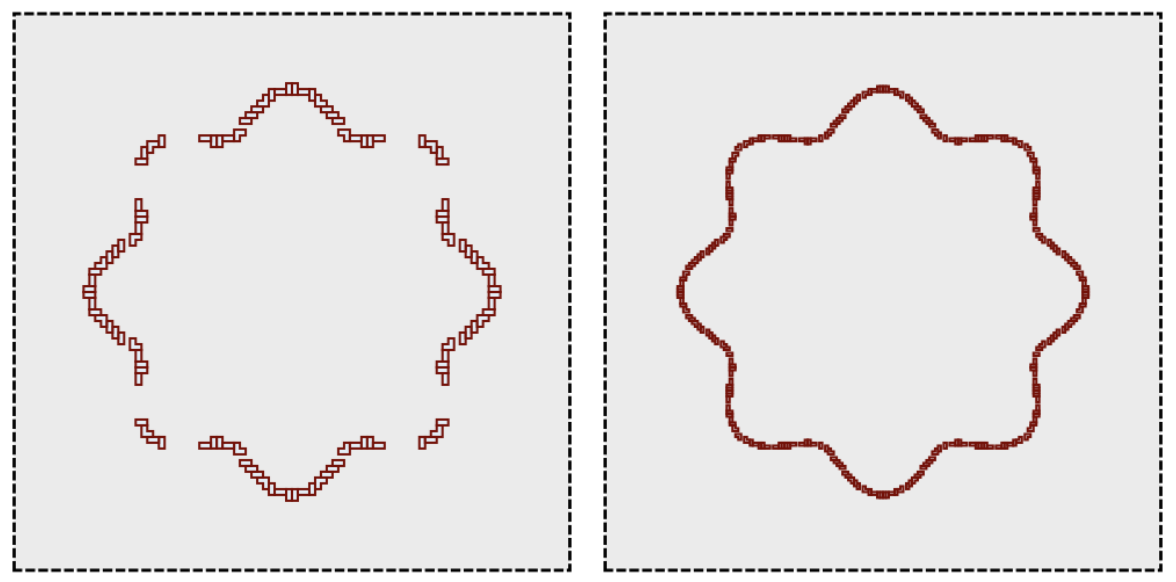

Figure 8: Agglomerated cells for the flower-like interface. Left: mesh used for the numerical comparisons $\left(h:=2^{-5}\right)$; right: mesh used to compute the reference solution $\left(h:=2^{-6}\right)$.

$\sigma_{y}$ at the two sensors $S_{1}$ and $S_{2}$ (the normalization factors are 0.036 for $\sigma_{x}$ at $S_{1}, 0.073$ for $\sigma_{y}$ at $S_{1}, 0.048$ for $\sigma_{x}$ at $S_{2}$, and 0.029 for $\sigma_{y}$ at $S_{2}$ ). In Table 5, we also include results for $k=1$ and a finer mesh so that the total number of coupled unknowns after static condensation is about the same as for $k=3$ (that is, $h=\frac{1}{90}$ for Newmark and $h=\frac{1}{45}$ for $\operatorname{SDIRK}(3,4)$ ). Comparing the corresponding errors with those obtained with $k=3$ (and even for $k=2$ ), we observe again the benefits of using a higher polynomial degree. The conclusion of this study is that we set the time refinement level to $l=8$ for Newmark and to $l=6$ for $\operatorname{SDIRK}(3,4)$, leading to relative errors below $2 \%$ (for Newmark) or $1 \%$ (for $\operatorname{SDIRK}(3,4)$ ) for the signals at both sensors. Finally, for the $\operatorname{ERK}(4)$ scheme, the time refinement level is dictated by the CFL condition and leads to $l=9$ and to relative errors below $1 \%$.

Table 6 reports some information on the CPU time for the three schemes. We employed the PARDISO sparse linear solver (Intel MKL library) for the direct solver and the Eigen library implementation of Bi-CGStab for the iterative solver with tolerance $10^{-10}$. The reported time values represent the total time per step employed on all the CPUs. The executions were performed on a 8-core CPU (2.7 GHz Quad-Core Intel Core i7) with LPDDR3 memory (16 GB $2133 \mathrm{MHz}$ ). All the implementations have been compiled with clang including the optimization level -03. The results from Table 6 should be considered as indicative of essential trends, as the current software is not optimized. The first observation is that the efficiency of RK schemes is higher than that of the Newmark scheme. Moreover, if direct solvers are allowed, the lowest over- 

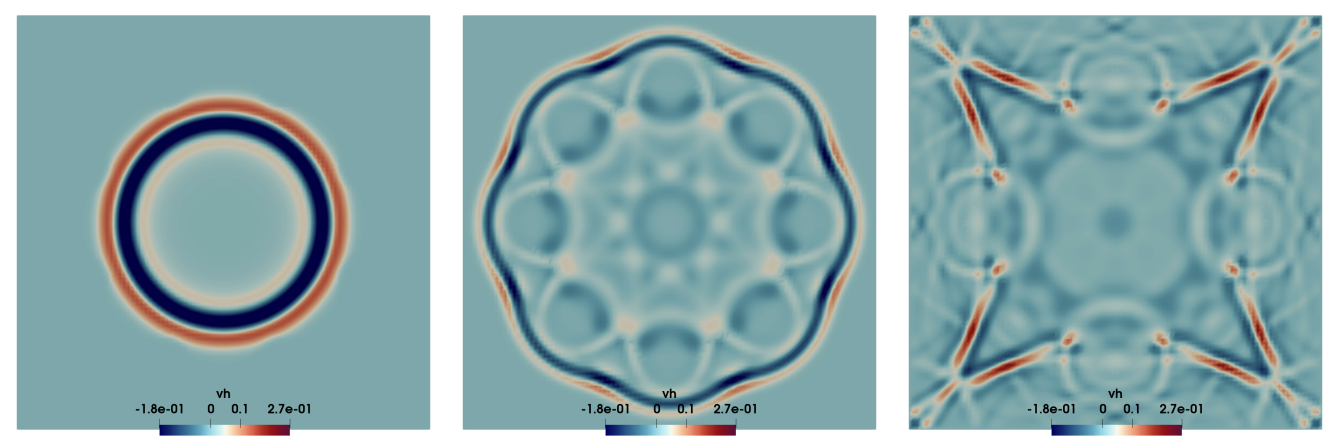

Figure 9: Flower-like test case: reference solution computing using $\operatorname{SDIRK}(3,4)$ time-stepping at the times $t \in\{0.25,0.5,1.0\}$.

all runtime (time/step multiplied by the number of steps needed to reach final time) is achieved by $\operatorname{SDIRK}(3,4)$, whereas if direct solvers are not allowed, the lowest overall runtime is achieved by $\operatorname{ERK}(4)$ despite the need to use a smaller time step due to the CFL condition. Finally, in Table 7 , we report for various schemes the CPU times [s] spent on the cell agglomeration procedure and the matrix assembly (we also indicate the total number of coupled unknowns after static condensation). The main observation is that the time spent on the cell agglomeration procedure is marginal with respect to the one spent on matrix assembly. Incidentally, we notice that an additional benefit of using a higher polynomial degree (while keeping approximately constant the total number of discrete unknowns) is that the cost of cell agglomeration becomes even more marginal.

\section{Conclusions}

We have devised unfitted HHO methods to approximate the acoustic wave equation in heterogeneous media. The unfitted methodology allows the interface to cut the cells in a general fashion, whereas the use of high polynomial orders for the space approximation is beneficial to improve solution accuracy. We have investigated the method theoretically by establishing an $H^{1}$-error estimate for the second-order formulation in time, leading to optimal decay rates of the error for smooth solutions. A thorough numerical study has been performed to assess the influence of the discretization of the geometry, the comparable accuracy between fitted and unfitted approaches in the case of a flat interface, and the moderate impact on the CFL condition for explicit time-stepping schemes when using the unfitted method with a relatively broad range of cell-agglomeration parameters. Finally, we have illustrated the capabilities of the proposed methodology in a test case where the interface has a more complex shape and we have performed a comparative study of the efficiency of the various time-stepping schemes. To conclude, we mention that the present study can be extended in a relatively straightforward manner to elastodynamics.

\section{Acknowledgments}

The authors would like to thank L. Guillot (CEA/DAM) for insightful discussions and CEA/DAM for partial financial support. EB was partially supported by the EPSRC grants EP/P01576X/1 and $\mathrm{EP} / \mathrm{P} 012434 / 1$. 

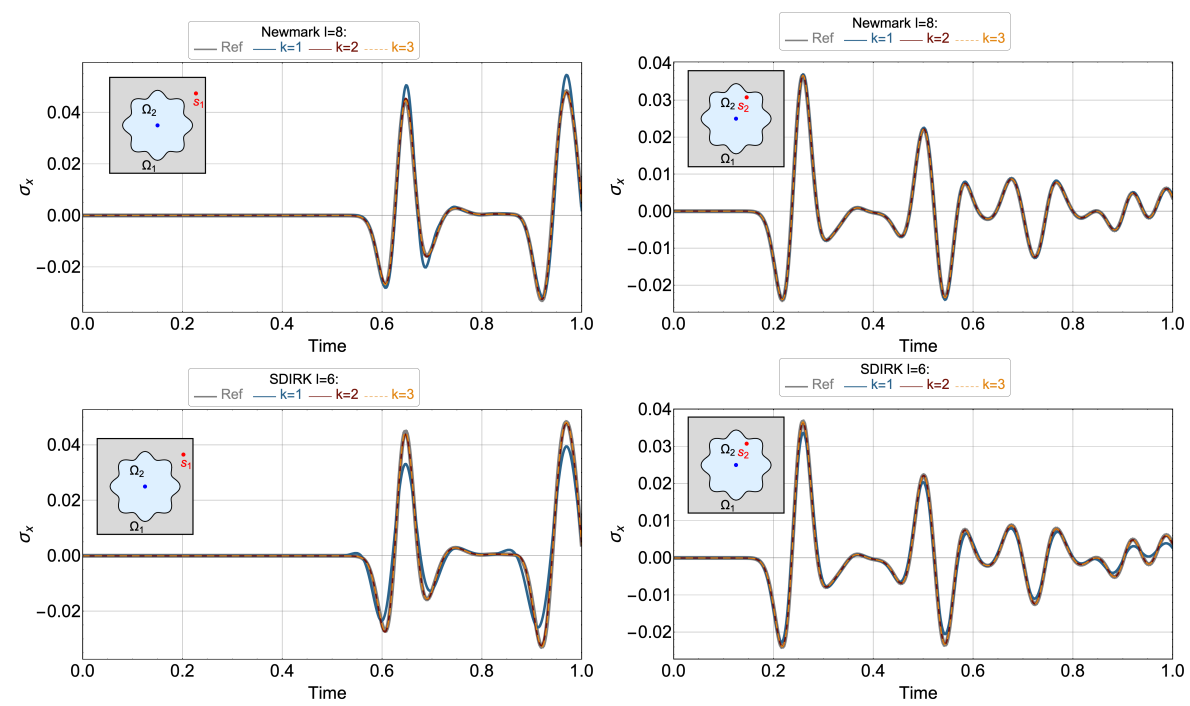

Figure 10: Flower-like test case. Comparison of the signal corresponding to $\sigma_{x}$ at the sensors $S_{1}$ (left column) and $S_{2}$ (right column) for the Newmark scheme (upper row, time refinement level $l=8$ ) and the $\operatorname{SDIRK}(3,4)$ scheme (bottom row, time refinement level $l=6$ ). The results obtained with polynomial orders $k \in\{1,2,3\}$ are compared to the reference solution.

\section{References}

[1] M. Abbas, A. Ern, and N. Pignet. Hybrid High-Order methods for finite deformations of hyperelastic materials. Comput. Mech., 62(4):909-928, 2018.

[2] S. Badia, F. Verdugo, and A. F. Martín. The aggregated unfitted finite element method for elliptic problems. Comput. Methods Appl. Mech. Engrg., 336:533-553, 2018.

[3] G. A. Baker. Error estimates for finite element methods for second order hyperbolic equations. SIAM J. Numer. Anal., 13(4):564-576, 1976.

[4] M. Botti, D. A. Di Pietro, and P. Sochala. A Hybrid High-Order method for nonlinear elasticity. SIAM J. Numer. Anal., 55(6):2687-2717, 2017.

[5] E. Burman. Ghost penalty. C. R. Math. Acad. Sci. Paris, 348(21-22):1217-1220, 2010.

[6] E. Burman, M. Cicuttin, G. Delay, and A. Ern. An unfitted hybrid high-order method with cell agglomeration for elliptic interface problems. SIAM J. Sci. Comput., 43(2):A859-A882, 2021.

[7] E. Burman, S. Claus, P. Hansbo, M. G. Larson, and A. Massing. CutFEM: discretizing geometry and partial differential equations. Internat. J. Numer. Methods Engrg., 104(7):472-501, 2015 .

[8] E. Burman, O. Duran, and A. Ern. Hybrid high-order methods for the acoustic wave equation in the time domain. Commun. Appl. Math. Comput., 2021. Available at https://hal archives-ouvertes fr/hal-02922702. 


\begin{tabular}{|c|c|c|c|c|c|c|c|}
\hline Newmark & \multicolumn{3}{|c|}{ Polynomial degree } & \multicolumn{3}{c|}{ Time ref. level } \\
\hline Variable and location & $k=1^{*}$ & $k=1$ & $k=2$ & $k=3$ & $l=6$ & $l=7$ & $l=8$ \\
\hline \hline$\sigma_{x}$ at $S_{1}$ & 8.157 & 15.40 & 3.954 & 1.640 & 10.15 & 3.817 & 1.640 \\
\hline$\sigma_{y}$ at $S_{1}$ & 7.433 & 16.24 & 3.977 & 1.835 & 11.35 & 4.272 & 1.835 \\
\hline$\sigma_{x}$ at $S_{2}$ & 1.817 & 2.154 & 1.506 & 1.421 & 7.301 & 3.115 & 1.421 \\
\hline$\sigma_{y}$ at $S_{2}$ & 2.688 & 2.946 & 1.469 & 1.421 & 7.303 & 3.116 & 1.421 \\
\hline
\end{tabular}

\begin{tabular}{|c|c|c|c|c|c|c|c|}
\hline SDIRK $(3,4)$ & \multicolumn{3}{|c|}{ Polynomial degree } & \multicolumn{3}{c|}{ Time ref. level } \\
\hline Variable and location & $k=1^{*}$ & $k=1$ & $k=2$ & $k=3$ & $l=4$ & $l=5$ & $l=6$ \\
\hline \hline$\sigma_{x}$ at $S_{1}$ & 5.698 & 25.05 & 2.996 & 0.3195 & 20.42 & 3.333 & 0.3195 \\
\hline$\sigma_{y}$ at $S_{1}$ & 7.279 & 31.72 & 4.189 & 0.5562 & 22.83 & 4.284 & 0.5562 \\
\hline$\sigma_{x}$ at $S_{2}$ & 1.459 & 8.683 & 0.4367 & 0.1281 & 12.81 & 1.646 & 0.1281 \\
\hline$\sigma_{y}$ at $S_{2}$ & 1.454 & 7.876 & 0.2282 & 0.1201 & 11.01 & 1.418 & 0.1201 \\
\hline
\end{tabular}

Table 5: Flower-like test case. Maximum relative errors (in \%) for the components $\sigma_{x}$ and $\sigma_{y}$ at the two sensors $S_{1}$ and $S_{2}$. Upper table: Newmark time-stepping; lower table: $\operatorname{SDIRK}(3,4)$ time-stepping. For each table, the largest $l$ is considered when varying $k$, and the largest $k$ when varying $l$. The column labeled $k=1^{*}$ uses a finer mesh so that the total number of coupled unknowns after static condensation is about the same as for $k=3\left(h=\frac{1}{90}\right.$ for Newmark and $h=\frac{1}{45}$ for $\left.\operatorname{SDIRK}(3,4)\right)$.

[9] E. Burman, O. Duran, A. Ern, and M. Steins. Convergence analysis of hybrid high-order methods for the wave equation. J. Sci. Comput., 87(3):Paper No. 91, 30, 2021.

[10] E. Burman and A. Ern. An unfitted hybrid high-order method for elliptic interface problems. SIAM J. Numer. Anal., 56(3):1525-1546, 2018.

[11] E. Burman, P. Hansbo, and M. G. Larson. Explicit Time Stepping for the Wave Equation using CutFEM with Discrete Extension. arXiv:2011.05386, 2020.

[12] M. Cicuttin, D. A. Di Pietro, and A. Ern. Implementation of discontinuous skeletal methods on arbitrary-dimensional, polytopal meshes using generic programming. J. Comput. Appl. Math., 344:852-874, 2018.

\begin{tabular}{|l|cc|ccc|cc|}
\hline scheme & sol. & stab. & time/step & steps & time & err. $S_{1}$ & err. $S_{2}$ \\
\hline \hline ERK $(4)$ & n/a & $\mathcal{O}(1)$ & 0.194 & 5120 & 495.6 & 0.16 & 0.02 \\
\hline \hline Newmark & dir. & $\mathcal{O}\left(h^{-1}\right)$ & 0.633 & 2560 & 1620.0 & 1.64 & 1.42 \\
SDIRK $(3,4)$ & dir. & $\mathcal{O}\left(h^{-1}\right)$ & 0.717 & 640 & 458.9 & 0.32 & 0.13 \\
SDIRK $(3,4)$ & iter. & $\mathcal{O}\left(h^{-1}\right)$ & 1.018 & 640 & 651.7 & 0.32 & 0.13 \\
\hline
\end{tabular}

Table 6: Flower-like test case. Comparative efficiency for the Newmark, $\operatorname{SDIRK}(3,4)$, and ERK(4) schemes. The column labeled "sol." reports whether a direct solver or an iterative solver is used, the column labeled "stab." the chocie for the stabilization weight $\tilde{\tau}_{\partial T}$, the column labeled "time/step" the CPU time per time-step [s], the column labeled "steps" the number of time-steps needed to reach the final time, the column labeled "time" the time/step multiplied by the number of steps, and the columns labeled "err" the relative maximum error for $\sigma_{x}$ at the two sensors over the time nodes. 


\begin{tabular}{|l|c|c|c|}
\hline & agglo & assembly & coupled dofs \\
\hline Newmark, $k=1, h=\frac{1}{90}$ & 4.64 & 21.20 & $291 \mathrm{k}$ \\
\hline Newmark, $k=3, h=\frac{1}{64}$ & 2.53 & 28.54 & $293 \mathrm{k}$ \\
\hline SDIRK $(3,4), k=1, h=\frac{1}{45}$ & 1.34 & 9.27 & $72 \mathrm{k}$ \\
\hline SDIRK $(3,4), k=3, h=\frac{1}{32}$ & 0.80 & 13.22 & $73 \mathrm{k}$ \\
\hline
\end{tabular}

Table 7: CPU times [s] for the cell agglomeration procedure and the matrix assembly, and total number of coupled unknowns after static condensation for various schemes.

[13] B. Cockburn, D. A. Di Pietro, and A. Ern. Bridging the hybrid high-order and hybridizable discontinuous Galerkin methods. ESAIM Math. Model Numer. Anal., 50(3):635-650, 2016.

[14] B. Cockburn, Z. Fu, A. Hungria, L. Ji, M. A. Sánchez, and F.-J. Sayas. Stormer-Numerov HDG methods for acoustic waves. J. Sci. Comput., 75(2):597-624, 2018.

[15] B. Cockburn and V. Quenneville-Bélair. Uniform-in-time superconvergence of the HDG methods for the acoustic wave equation. Math. Comp., 83(285):65-85, 2014.

[16] G. C. Cohen. Higher-Order Numerical Methods for Transient Wave Equations. Springer Berlin Heidelberg, 2002.

[17] D. A. Di Pietro and A. Ern. A hybrid high-order locking-free method for linear elasticity on general meshes. Comput. Meth. Appl. Mech. Engrg., 283:1-21, 2015.

[18] D. A. Di Pietro, A. Ern, and S. Lemaire. An arbitrary-order and compact-stencil discretization of diffusion on general meshes based on local reconstruction operators. Comput. Meth. Appl. Math., 14(4):461-472, 2014.

[19] D. A. Dunavant. High degree efficient symmetrical Gaussian quadrature rules for the triangle. Int. J. Numer. Methods Eng., 21(6):1129-1148, 1985.

[20] T. Dupont. $L^{2}$-estimates for Galerkin methods for second order hyperbolic equations. SIAM J. Numer. Anal., 10:880-889, 1973.

[21] L. C. Evans. Partial differential equations, volume 19 of Graduate Studies in Mathematics. American Mathematical Society, Providence, RI, 1998.

[22] R. S. Falk and G. R. Richter. Explicit finite element methods for symmetric hyperbolic equations. SIAM J. Numer. Anal., 36(3):935-952, 1999.

[23] R. Griesmaier and P. Monk. Discretization of the wave equation using continuous elements in time and a hybridizable discontinuous Galerkin method in space. J. Sci. Comput., 58(2):472498, 2014.

[24] M. J. Grote, A. Schneebeli, and D. Schötzau. Discontinuous Galerkin finite element method for the wave equation. SIAM J. Numer. Anal., 44(6):2408-2431, 2006.

[25] A. Johansson and M. G. Larson. A high order discontinuous Galerkin Nitsche method for elliptic problems with fictitious boundary. Numer. Math., 123(4):607-628, 2013.

[26] C. Lehrenfeld. Hybrid Discontinuous Galerkin methods for solving incompressible flow problems. PhD thesis, Rheinisch-Westfälische Technische Hochschule (RWTH) Aachen, 2010. 
[27] C. Lehrenfeld and J. Schöberl. High order exactly divergence-free hybrid discontinuous Galerkin methods for unsteady incompressible flows. Comput. Methods Appl. Mech. Engrg., 307:339-361, 2016.

[28] P. Monk and G. R. Richter. A discontinuous Galerkin method for linear symmetric hyperbolic systems in inhomogeneous media. J. Sci. Comput., 22/23:443-477, 2005.

[29] N. C. Nguyen, J. Peraire, and B. Cockburn. High-order implicit hybridizable discontinuous Galerkin methods for acoustics and elastodynamics. J. Comput. Phys., 230(10):3695-3718, 2011.

[30] M. A. Sánchez, C. Ciuca, N. C. Nguyen, J. Peraire, and B. Cockburn. Symplectic Hamiltonian HDG methods for wave propagation phenomena. J. Comput. Phys., 350:951-973, 2017.

[31] S. Schoeder, S. Sticko, G. Kreiss, and M. Kronbichler. High-order cut discontinuous Galerkin methods with local time stepping for acoustics. Internat. J. Numer. Methods Engrg., 121(13):2979-3003, 2020.

[32] M. Stanglmeier, N. C. Nguyen, J. Peraire, and B. Cockburn. An explicit hybridizable discontinuous Galerkin method for the acoustic wave equation. Comput. Methods Appl. Mech. Engrg., 300:748-769, 2016.

[33] S. Sticko and G. Kreiss. A stabilized Nitsche cut element method for the wave equation. Comput. Methods Appl. Mech. Engrg., 309:364-387, 2016.

[34] S. Sticko and G. Kreiss. Higher order cut finite elements for the wave equation. J. Sci. Comput., 80(3):1867-1887, 2019.

[35] S. Sticko, G. Ludvigsson, and G. Kreiss. High-order cut finite elements for the elastic wave equation. Adv. Comput. Math., 46(3):Paper No. 45, 28, 2020.

[36] C. C. Stolk. On the modeling and inversion of seismic data. PhD thesis, Utrecht University, The Netherlands, 2000. http://dspace.library.uu.nl/handle/1874/855.

[37] S. Terrana, J. P. Vilotte, and L. Guillot. A spectral hybridizable discontinuous Galerkin method for elastic-acoustic wave propagation. Geophysical Journal International, 213(1):574-602, 2017.

[38] J. Wang and X. Ye. A weak Galerkin finite element method for second-order elliptic problems. J. Comput. Appl. Math., 241:103-115, 2013. 